\title{
Integrated Supply Chain Cooperative Inventory Model with Payment Period Being Dependent on Purchasing Price under Defective Rate Condition
}

\author{
Ming-Feng Yang, ${ }^{1}$ Jun-Yuan Kuo, ${ }^{2}$ Wei-Hao Chen, ${ }^{3}$ and Yi Lin ${ }^{4}$ \\ ${ }^{1}$ Department of Transportation Science, National Taiwan Ocean University, Keelung City 202, Taiwan \\ ${ }^{2}$ Department of International Business, Kainan University, Taoyuan 338, Taiwan \\ ${ }^{3}$ Department of Shipping and Transportation Management, National Taiwan Ocean University, Keelung City 202, Taiwan \\ ${ }^{4}$ Graduate Institute of Industrial and Business Management, National Taipei University of Technology, No. 1, \\ Sec. 3, Zhongxiao E. Road, Taipei City 106, Taiwan
}

Correspondence should be addressed to Ming-Feng Yang; yang60429@mail.ntou.edu.tw

Received 18 August 2014; Revised 7 November 2014; Accepted 18 November 2014

Academic Editor: Mu-Chen Chen

Copyright (C) 2015 Ming-Feng Yang et al. This is an open access article distributed under the Creative Commons Attribution License, which permits unrestricted use, distribution, and reproduction in any medium, provided the original work is properly cited.

\begin{abstract}
In most commercial transactions, the buyer and vendor may usually agree to postpone payment deadline. During such delayed period, the buyer is entitled to keep the products without having to pay the sale price. However, the vendor usually hopes to receive full payment as soon as possible, especially when the transaction involves valuable items; yet, the buyer would offer a higher purchasing price in exchange of a longer postponement. Therefore, we assumed such permissible delayed period is dependent on the purchasing price. As for the manufacturing side, defective products are inevitable from time to time, and not all of those defective products can be repaired. Hence, we would like to add defective production and repair rate to our proposed model and discuss how these factors may affect profits. In addition, holding cost, ordering cost, and transportation cost will also be considered as we develop the integrated inventory model with price-dependent payment period under the possible condition of defective products. We would like to find the maximum of the joint expected total profit for our model and come up with a suitable inventory policy accordingly. In the end, we have also provided a numerical example to clearly illustrate possible solutions.
\end{abstract}

\section{Introduction}

Inventory occurs in every stage of the supply chain; therefore, managing inventory in an effective and efficient way becomes a significant task for managers in the course of supply chain management (SCM). Fogarty [1] pointed out that the purpose of inventory is to retrieve demand and supply in an uncertain environment. Frankel [2] considered supply chain to be closely related to controlling and preserving stocks. A good inventory policy should contain a right venue to order, to manufacture, and to distribute accurate supply quantities at the right moment which will then store inventory at the right place to minimize total cost. Another reason for the need to collaborate with other members in the supply chain is to remain competitive. Better collaboration with customers and suppliers will not only provide better service but also reduce costs [3]. Beheshti [4] considered inventory policy as the key to affect conditions during the supply chain, and applying inappropriate inventory policy would result in great loss. Therefore, it is crucial for SCM practice to generate suitable inventory policy. Since the EOQ model proposed by Harris [5] and researchers as well as practitioners have shown interest in optimal inventory policy, Harris [5] focused on inventory decisions of individual firms; yet from the SCM perspective, collaborating closely with members of the supply chain is certainly necessary. Goyal [6] is the first researcher to point out the importance of performance when integrating a supplier and a customer's inventory policies. The single-supplier single-customer model showed the total relevant cost reduction compared with traditional independent inventory strategy. Jammernegg and Reiner [7] pointed out that effective inventory management can enhance 
the value of the full supply chain. Olson and Xie [8] proposed purchasers and sellers should have a common inventory system when they cooperate with each other. Since supply chain is formed with multiple firms, focusing on a vendor and a buyer's inventory problem is not sufficient. In other words, multiechelon inventory problem is one of the leading issues in SCM. Huang et al. [9] developed an inventory model as three-level dynamic noncooperative game by using the Nash equilibrium. Giannoccaro and Pontrandolfo [10] developed an inventory forecast for three-echelon supply chain to minimize the joint total cost. Cárdenas-Barrón et al. [11] made complements to some shortcomings in the model proposed by Sana [12] and then introduced alternative algorithm to obtain shorter CPU time and fewer total cost [3]. Sana [12] coordinated production and inventory decisions across the supplier, the manufacture, and the customer to maximize the total expected profits. Chung et al. [13] combined deteriorating items with two levels of trade credit under three-layer condition in the supply chain system. A new economic production quantity (EPQ) inventory is then proposed to minimize the total cost. Yang and Tseng [14] assumed that defective products occurred in the supplier and the manufacturer stage, and then backorder is allowed to develop a three-echelon inventory model. Permissible delay in payments and controllable lead time are also considered in the model.

Yield rate is an important factor in manufacturing industry. Production can be imperfect, which may have resulted from insufficient process control, wrongly planned maintenance, inadequate work instructions, or damages during handling (Rad et al., [15]). High defective rate will increase not only production costs but also inspecting costs and repair costs, which may likely cause shortage during the process. In early researches, defective production was rarely considered in economic ordering quantity (EOQ) model; however, defective production is a common condition in real practice. Schwaller [16] added fixed defective rate and inspecting costs to the traditional EOQ model. Paknejad et al. [17] developed an imperfect inventory model under random demands and fixed lead time. Liu and Yang [18] developed an imperfect inventory model which included good products, repairable products, and scrap to maximize the joint total profits. Salameh and Jaber [19] indicated that all products should be divided into good products and defective products; they found that EOQ will increase as defective products increase. Eroglu and Ozdemir [20] extended Salameh and Jaber's [19] model, who indicated how defective rate affects economic production quantity (EPQ) with defective products and permissible shortage. All defective products can be inspected and sold separately from good products. Pal et al. [21] developed a three-layer integrated production-inventory model considering out-ofcontrol quality may occur in the supplier and manufacturer stage. The defective products are reworked at a cost after the regular production time. Using Stakelberg's approach, we can see that the integrated expected average profit was being compared with the total expected average profits. Sarkar et al. [22] extended such work and developed three inventory models considering that the proportion of products could follow different probability distribution: uniform, triangular, and beta. The models allowed planned backorders and the defective products to be reworked [23]. The comparison table was made to show that the minimum cost is obtained in the case of triangular distribution. Soni and Patel [24] assumed that an arrival order lot may contain defective items, and the number of defective items is a random variable which follows beta distribution in a numerical example. The demand is sensitive to retail price, and the production rate will react to demand.

Recently, permissible delay in payments has become a common commercial strategy between the vendor and the buyer. It will bring additional interests or opportunity costs to each other as permissible delayed period varies; hence, delayed period is a critical issue that researchers should consider when developing inventory models. In traditional EOQ assumptions, the buyer has to pay upon product delivery; however, in actual business transactions, the vendor usually gives a fixed delayed period to reduce the stress of capital. During such period, the buyer can make use of the products without having to pay to the vendor; both parties can earn extra interests from sales. Goyal [25] developed an EOQ model with delays in payments. Two situations were discussed in the research: (1) time interval between successive orders was longer than or equal to permissible delay in settling accounts; (2) time interval between successive orders was shorter than permissible delay in settling accounts. Aggarwal and Jaggi [26] quoted Goyal's [25] assumptions to develop a deteriorating inventory model under fixed deteriorating rate. Jamal et al. [27] extended Aggarwal and Jaggi's [26] model and added shortage condition. Teng [28] also amended Goyal's [25] EOQ model and acquired two conclusions. (1) The EOQ decreases and the order cycle period shortens. It is different from Goyal's [25] conclusion. (2) If the supplier wants to decrease the stocks, the supplier has to set higher interest rate to the retailer unpaid payments after the payment periods are overdue, but this will cause the EOQ to be higher than traditional EOQ model. Huang et al. [29] developed a vendor-buyer inventory model with order processing cost reduction and permissible delay in payments. They considered applying information technologies to reduce order processing cost as long as the vendor and the buyer are willing to pay additional investment costs. They also showed that Ha and Kim's [30] model is actually a special case. Lou and Wang [31] extended Huang's [32] integrated inventory model which discussed the relationship between the vendor and the buyer in trade credit financing. They relaxed the assumption that the buyer's interest earned is always less than or equal to the interests charged. They also established a discrimination term to determine whether the buyer's replenishment cycle time is less than the permissible delay period. Li et al. [33] extended the model of Meca et al. [34] by adding permissible payment delays into the corresponding inventory game. They also showed that the core of the inventory game is nonempty and the grand coalition is stable in a myopic perspective; therefore, largest consistent set (LCS) is applied to improve the grand coalition. While most of EOQ models are considered with infinite replenishment rate, Sarkar et al. [35] developed EOQ model for various types of 
time-dependent demand when delay in payment and price discount are permitted by suppliers in order to obtain the optimal cycle time with finite replenishment rate.

The main purpose of this paper is to maximize the expected joint total profits. Based on Yang and Tseng's [14] model, we also considered the fact that some defective products can be repaired. Furthermore, we proposed functions between purchasing costs and permissible delayed payment period to balance the opportunity costs and interests income when we promote cooperation. We first defined the parameters and assumptions in Section 2, and then we started to develop the integrated inventory model in Section 3. In Section 4, we tried to solve the model to get the optimal solution. A series of numerical examples would be discussed to observe the variations of decision variables by changing parameters in Section 5. In the end, we summarized the variation and present conclusions.

\section{Notations and Assumptions}

We first develop a three-echelon inventory model with repairable rate and include permissible delay in payments dependent on sale price. The expected joint total annual profits of the model can be divided into three parts: the annual profit of the supplier, the manufacturer, and the retailer. We then observe how purchasing cost may affect permissible delayed period, EOQ, the number of delivery per production run, and the expected joint total annual profits under different manufacturer's production rate and defective rate.

2.1. Notations. To establish the mathematical model, the following notations and assumptions are used. The notations are shown as follows.

\section{The Parameters and the Decision Variable}

$Q_{i}$ : Economic delivery quantity of the $i$ th model, $i=$ $1,2,3,4$, a decision variable

$n_{i}$ : The number of lots delivered in a production cycle from the manufacturer to the retailer of $i$ th model, $i=$ $1,2,3,4$, a positive integer and a decision variable.

\section{(i) Supplier Side}

$C_{s}$ : Supplier's purchasing cost per unit

$A_{s}$ : Supplier's ordering cost per order

$h_{s}$ : Supplier's annual holding cost per unit

$I_{\mathrm{sp}}$ : Supplier's opportunity cost per dollar per year

$I_{\mathrm{se}}$ : Supplier's interest earned per dollar per year.

\section{(ii) Manufacturer Side}

$P$ : Manufacturer's production rate

$X$ : Manufacturer's permissible delayed period

$C_{m}$ : Manufacturer's purchasing cost per unit

$A_{m}$ : Manufacturer's ordering cost per order
$Z$ : The probability of defective products from manufacturer

$R$ : The probability of defective products can be repaired

$W$ : Manufacturer's inspecting cost per unit

$C_{\mathrm{rm}}$ : Manufacturer's repair cost per unit

$G$ : Manufacturer's scrap cost per unit

$t_{s}$ : The time for repairing all defective products at manufacturer

$F_{m}$ : Manufacturer's transportation cost per shipment

$h_{m}$ : Manufacturer's annual holding cost per unit

$L_{m}$ : The length of lead time of manufacturer

$I_{\mathrm{mp}}$ : Manufacturer's opportunity cost per dollar per year

$I_{\text {me }}$ : Manufacturer's interest earned per dollar per year.

(iii) Retailer Side

$D$ : Average annual demand per unit time

$Y$ : Retailer's permissible delayed period

$P_{r}$ : Retailer's selling price per unit

$C_{r}$ : Retailer's purchasing cost per unit

$A_{r}$ : Retailer's ordering cost per order

$F_{r}$ : Retailer's transportation cost per shipment

$h_{r}$ : Retailer's annual holding cost per unit

$L_{r}$ : The length of lead time of retailer

$I_{\mathrm{rp}}$ : Retailer's opportunity cost per dollar per year

$I_{\mathrm{re}}$ : Retailer's interest earned per dollar per year

$\mathrm{TP}_{s}$ : Supplier's total annual profit

$\mathrm{TP}_{m}$ : Manufacturer's total annual profit

$\mathrm{TP}_{r}$ : Retailer's total annual profit

EJTP $_{i}$ : The expected joint total annual profit, $i=$ $1,2,3,4$.

Note. " $i$ " represents four different cases due to the relationship of lead time and permissible payment period of manufacturer and the relationship of lead time and permissible payment period of retailer. We will have more detailed discussions in Section 3.

\subsection{Assumptions}

(1) This supply chain system consists of a single supplier, a single manufacturer, and a single retailer for a single product.

(2) Economic delivery quantity multiplied by the number of deliveries per production run is economic order quantity (EOQ).

(3) Shortages are not allowed. 
(4) The sale price must not be less than the purchasing cost at any echelon, $P_{r} \geq C_{r} \geq C_{m} \geq C_{s}$.

(5) Defective products only happened in the manufacturer and can be inspected and separated into repairable products and scrap immediately.

(6) Scrap cannot be recycled, so the manufacturer has to pay to throw away.

(7) The seller provides a permissible delayed period ( $X$ and $Y$ ). During the period, the purchaser keeps selling the products and earning the interest by selling revenue. The purchaser pays to the seller at the end of the time period. If the purchaser still has stocks, it will bring capital cost.

(8) The lead time of manufacturer is equal to the cycle time $\left(L_{m}=n Q / D\right)$. The lead time of supplier is equal to the cycle time $\left(L_{r}=Q / D\right)$.

(9) The purchasing cost is in inverse to the permissible delayed period. It means that the cheaper the purchasing cost, the longer the permissible delayed period.

(10) The time horizon is infinite.

\section{Model Formulation}

In this section, we have discussed the model of supplier, manufacture, and retailer, and we combined them all into an integrated inventory model. We extended Yang and Tseng's [14] research to compute opportunity costs and interests income. Finally, we used the function between purchasing costs and the permissible delayed payment period to discuss and observe the variation of the expected joint total annual profits.

3.1. The Supplier's Total Annual Profit. In each production run, the supplier's revenue includes sales revenue and interest income; the supplier's includes ordering cost, holding cost, and opportunity cost. Under the condition of permissible delay in payments, if the payment time of the manufacturer $(X)$ is longer than the lead time of the manufacturer $\left(L_{m}\right)$, it will bring additional interests income based on its interest rate $\left(I_{\text {me }}\right)$ to the manufacturer. On the other hand, it causes the supplier to pay additional opportunity cost based on its interest rate $\left(I_{\mathrm{sp}}\right)$. If the payment time of the manufacturer $(X)$ is shorter than the lead time of the manufacturer $\left(L_{m}\right)$, it will bring not only additional interests income but also the opportunity costs based on its interest rate $\left(I_{\mathrm{me}}\right.$ and $\left.I_{\mathrm{sp}}\right)$ separately to the manufacturer because of the rest of stocks; however, it causes the supplier to pay additional opportunity costs but gains additional interests income based on its interest rate $\left(I_{\mathrm{sp}}\right.$ and $\left.I_{\mathrm{se}}\right)$ separately.

Before we start to establish the inventory model, we have to discuss how defective rate $(Z)$ and repair rate $(R)$ can affect yield rate. In each production run, the manufacturer outputs defective products because of the imperfect production line. In other words, yield rate is $(1-Z)$. There is fixed proportion to repair these defective products, which means that the proportion of repaired products is $(Z R)$. Since the repaired

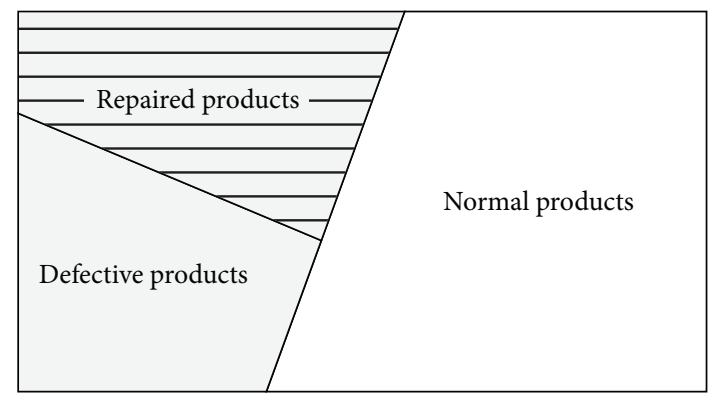

FIGURE 1: Three kinds of products in the production run.

products are counted in the yield products, we have to revise yield rate by adding the proportion of repaired products. Figure 1 showed the relationship of defective rate, repair rate, and yield rate. So revised yield rate is $(1-Z(1-R))$. In order to satisfy the demand in each production run, the manufacturer will request the supplier to deliver $(n Q) /[1-Z(1-R)]$.

Figure 2 showed the supplier, manufacturer, and retailer's inventory level. As mentioned before, the retailer needs ( $n Q)$ to satisfy the demand, while the manufacturer produces $(n Q) /[1-Z(1-R)]$ due to defective rate and repair rate, and the supplier would need to prepare $(n Q) /[1-Z(1-R)]$ to prevent storage.

Case $1\left(L_{m}<X\right)$. If $L_{m}<X$, the manufacturer will earn interests income, but the manufacturer's interests income will be transferred into opportunity costs for the supplier (see Figure 3). Consider the following.

(i) Sales revenue $=D\left(C_{m}-C_{s}\right) /(1-Z(1-R))$.

(ii) Ordering cost $=A_{s} D / n_{i} Q_{i}$.

(iii) Holding cost $=h_{s} D n_{i} Q_{i} / 2 P[1-Z(1-R)]^{2}$.

(iv) Transfer opportunity cost $=C_{s} I_{\mathrm{sp}}\left(2 D X-n_{i} Q_{i}\right) / 2[1-$ $Z(1-R)]$.

Thus, $\mathrm{TP}_{s 1}$ is given by

$\mathrm{TP}_{s 1}=$ sales revenue - ordering cost - holding cost

- transfer opportunity cost

$$
\begin{aligned}
= & \frac{D\left(C_{m}-C_{s}\right)}{1-Z(1-R)}-\frac{A_{s} D}{n_{i} Q_{i}}-\frac{h_{s} D n_{i} Q_{i}}{2 P[1-Z(1-R)]^{2}} \\
& -\frac{C_{s} I_{\text {sp }}\left(2 D X-n_{i} Q_{i}\right)}{2[1-Z(1-R)]} .
\end{aligned}
$$

Case $2\left(L_{m} \geq X\right)$. If $L_{m} \geq X$, the manufacturer will not only earn interests income but also pay the opportunity costs due to the rest of stocks. The manufacturer's interests income and opportunity costs will be transferred into opportunity costs and interests income for the supplier (see Figure 4). Consider the following.

(i) Transfer opportunity cost $=C_{s} I_{\mathrm{sp}}\left(2 D X-n_{i} Q_{i}\right) / 2[1-$ $Z(1-R)]$. 


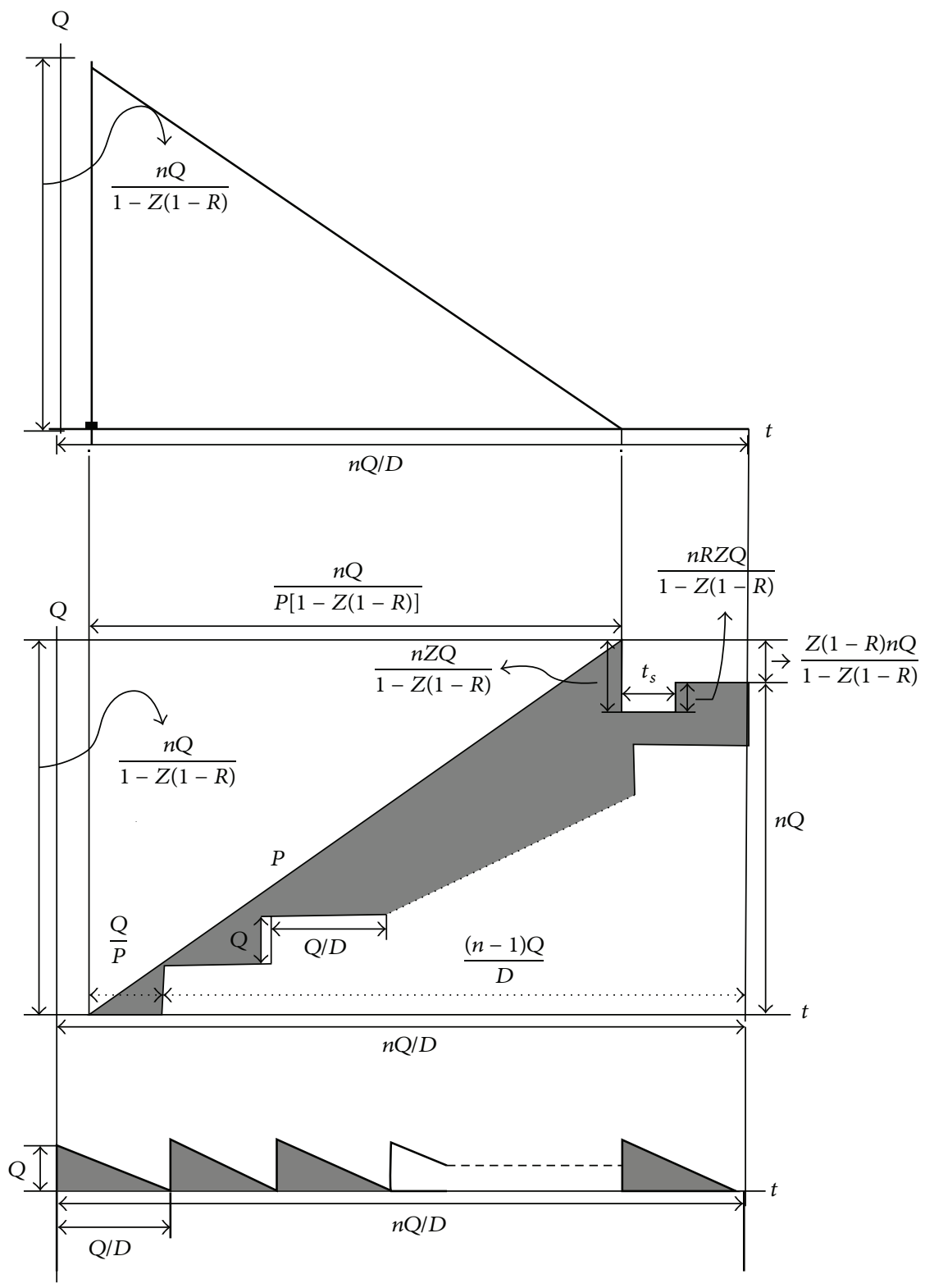

FIGURE 2: The inventory pattern for the three firms.

(ii) Transfer interest income $=C_{m} I_{\mathrm{se}}\left(n_{i} Q_{i}-D X\right)^{2} / 2 n[1-$ $Z(1-R)] Q_{i}$.

Thus, $\mathrm{TP}_{s 2}$ is given by

$\mathrm{TP}_{s 2}=$ sales revenue - ordering cost - holding cost

- transfer opportunity cost + interest income

$$
\begin{gathered}
=\frac{D\left(C_{m}-C_{s}\right)}{1-Z(1-R)}-\frac{A_{s} D}{n_{i} Q_{i}}-\frac{h_{s} D n_{i} Q_{i}}{2 P[1-Z(1-R)]^{2}} \\
=-\frac{C_{s} I_{\text {sp }}\left(2 D X-n_{i} Q_{i}\right)}{2[1-Z(1-R)]}+\frac{C_{m} I_{\text {se }}\left(n_{i} Q_{i}-D X\right)^{2}}{2 n[1-Z(1-R)] Q_{i}} .
\end{gathered}
$$

3.2. The Manufacturer's Total Annual Profit. In each production run, the manufacturer's revenue includes sales revenue and interests income; the manufacturer's cost includes ordering costs, holding costs, transportation costs, inspecting costs, repair costs, scrap costs, and opportunity costs. We have discussed the relationship between the lead time of the manufacturer $\left(L_{m}\right)$ and the payment time of the manufacturer $(X)$. This relationship can be also used to discuss the retailer's lead time $\left(L_{r}\right)$ and the payment time $(Y)$; therefore, the manufacturer's total annual profit has four different cases. In the middle of Figure 2 is the manufacturer's inventory level which has been the effect of defective rate and repair rate.

Case $1\left(L_{m}<X, L_{r}<Y\right)$. If $L_{m}<X$ and $L_{r}<Y$, both the manufacturer and the retailer will earn interests income, but the retailer's interests income will be transferred 


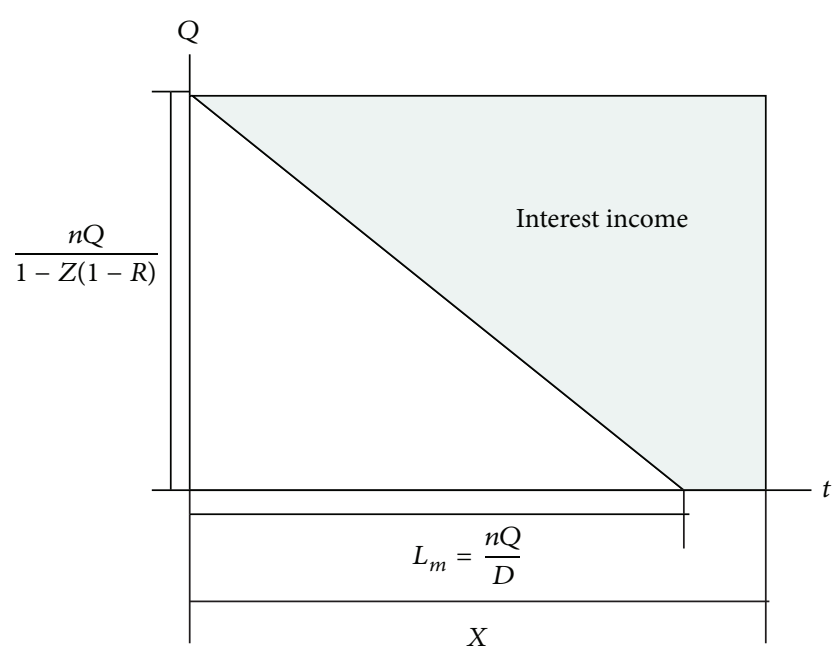

FIGURE 3: $L_{m}<X$.

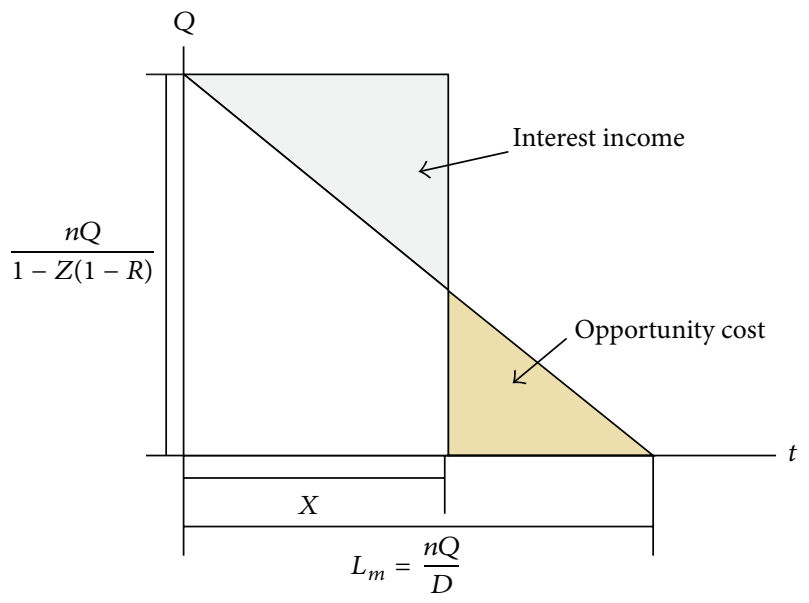

FiguRE 4: $L_{m} \geq X$.

into opportunity costs for the manufacturer. Consider the following.

(i) Sales revenue $=D\left[C_{r}-C_{m} /(1-Z(1-R))\right]$.

(ii) Ordering cost $=A_{m} D / n_{i} Q_{i}$.

(iii) Holding cost $=h_{m} D\left\{Q_{i}\left[\left(n_{i}-1\right) / 2 D+\{1-2[1-Z(1-\right.\right.$ $\left.\left.R)]\} n_{i} / 2 P[1-Z(1-R)]^{2}+1 / P\right]-t_{s} Z R n_{i} /(1-Z(1-R))\right\}$.

(iv) Transportation cost $=F_{m} D / n_{i} Q_{i}$.

(v) Inspecting cost $=W D /(1-Z(1-R))$.

(vi) Repair cost $=W D /(1-Z(1-R))$.

(vii) Scrap cost $=G Z(1-R) D /(1-Z(1-R))$.

(viii) Interest income $=C_{r} I_{\text {me }}\left(2 D X-n_{i} Q_{i}\right) / 2[1-Z(1-R)]$.

(ix) Transfer opportunity cost $=C_{m} I_{\mathrm{mp}}\left(D Y-Q_{i} / 2\right)$.
Thus, $\mathrm{TP}_{m 1}$ is given by

$\mathrm{TP}_{m 1}$

$=$ sales revenue - ordering cost - holding cost

- transportation cost - inspecting cost - repair cost

- scrap cost + interest income

- transfer opportunity cost

$$
\begin{gathered}
=D\left[C_{r}-\frac{C_{m}}{1-Z(1-R)}\right]-\frac{A_{m} D}{n_{i} Q_{i}} \\
-h_{m} D\left\{Q_{i}\left[\frac{n_{i}-1}{2 D}+\frac{\{1-2[1-Z(1-R)]\} n_{i}}{2 P[1-Z(1-R)]^{2}}+\frac{1}{P}\right]\right. \\
\left.\quad-\frac{t_{s} Z R n_{i}}{1-Z(1-R)}\right\} \\
-\frac{F_{m} D}{n_{i} Q_{i}}-\frac{D\left[W+C_{\mathrm{rm}} Z R+G Z(1-R)\right]}{1-Z(1-R)} \\
+\frac{C_{r} I_{\mathrm{me}}\left(2 D X-n_{i} Q_{i}\right)}{2[1-Z(1-R)]}-C_{m} I_{\mathrm{mp}}\left(D Y-\frac{Q_{i}}{2}\right) .
\end{gathered}
$$

Case $2\left(L_{m}<X, L_{r}<Y\right)$. If $L_{m}<X$ and $L_{r} \geq Y$, the manufacturer will earn interests income while the retailer will not due to the rest of stocks, but the retailer's interests income and opportunity costs will be transferred into opportunity costs and interests income for the manufacturer:

$$
\text { Interest income }=\frac{C_{r} I_{\mathrm{me}}\left(2 D X-n_{i} Q_{i}\right)}{2[1-Z(1-R)]} .
$$

Consider the following.

(i) Transfer opportunity cost $=C_{m} I_{\mathrm{mp}}(D Y)^{2} / 2 Q_{i}$.

(ii) Transfer interest income $=C_{r} I_{\mathrm{me}}\left(Q_{i}-D Y\right)^{2} / 2 Q_{i}$.

Thus, $\mathrm{TP}_{m 2}$ is given by

$\mathrm{TP}_{m 2}$

$=$ sales revenue - ordering cost - holding cost

- transportation cost - inspecting cost - repair cost

- scrap cost + interest income

- transfer opportunity cost + transfer interest income

$$
\begin{aligned}
=D\left[C_{r}-\frac{C_{m}}{1-Z(1-R)}\right]-\frac{A_{m} D}{n_{i} Q_{i}} \\
-h_{m} D\left\{Q_{i}\left[\frac{n_{i}-1}{2 D}+\frac{\{1-2[1-Z(1-R)]\} n_{i}}{2 P[1-Z(1-R)]^{2}}+\frac{1}{P}\right]\right. \\
\left.-\frac{t_{s} Z R n_{i}}{1-Z(1-R)}\right\}
\end{aligned}
$$




$$
\begin{aligned}
& -\frac{F_{m} D}{n_{i} Q_{i}}-\frac{D\left[W+C_{\mathrm{rm}} Z R+G Z(1-R)\right]}{1-Z(1-R)} \\
& +\frac{C_{r} I_{\mathrm{me}}\left(2 D X-n_{i} Q_{i}\right)}{2[1-Z(1-R)]}-\frac{C_{m} I_{\mathrm{mp}}(D Y)^{2}}{2 Q_{i}} \\
& +\frac{C_{r} I_{\mathrm{me}}\left(Q_{i}-D Y\right)^{2}}{2 Q_{i}} .
\end{aligned}
$$

Case $3\left(L_{m} \geq X, L_{r}<Y\right)$. If $L_{m} \geq X$ and $L_{r}<Y$, the manufacturer will not earn interests income but also pay opportunity costs, and the retailer will earn interests income but such income will be transferred into opportunity costs for the manufacturer. Consider the following.

(i) Opportunity cost $=C_{m} I_{\mathrm{mp}}\left(n_{i} Q_{i}-D X\right)^{2} / 2[1-Z(1-$ R)] $n_{i} Q_{i}$.

(ii) Interest income $=C_{r} I_{\mathrm{me}}(D X)^{2} / 2[1-Z(1-R)] n_{i} Q_{i}$.

(iii) Transfer opportunity cost $=C_{m} I_{\mathrm{mp}}\left(D Y-Q_{i} / 2\right)$.

Thus, $\mathrm{TP}_{m 3}$ is given by

$$
\begin{aligned}
\mathrm{TP}_{m 3} & \text { sales revenue }- \text { ordering cost }- \text { holding cost } \\
& - \text { transportation cost }- \text { inspecting cost }- \text { repair cost } \\
& - \text { scrap cost }- \text { opportunity cost }+ \text { interest income } \\
& - \text { transfer opportunity cost } \\
= & D\left[C_{r}-\frac{C_{m}}{1-Z(1-R)}\right]-\frac{A_{m} D}{n_{i} Q_{i}} \\
& -h_{m} D\left\{Q_{i}\left[\frac{n_{i}-1}{2 D}+\frac{\{1-2[1-Z(1-R)]\} n_{i}}{2 P[1-Z(1-R)]^{2}}+\frac{1}{P}\right]\right. \\
& -\frac{F_{m} D}{n_{i} Q_{i}}-\frac{D\left[W+C_{\mathrm{rm}} Z R+G Z(1-R)\right]}{1-Z(1-R)} \\
& \left.-\frac{C_{m} I_{\mathrm{mp}}}{2\left[1-Z\left(n_{i} Q_{i}-D X\right)^{2}\right.}+\frac{C_{r} I_{\mathrm{me}}(D X)^{2}}{2[1-Z)] n_{i} Q_{i}}\right\} \\
& -C_{m} I_{\mathrm{mp}}\left(D Y-\frac{Q_{i}}{2}\right) \cdot \\
& -\frac{R(1-R)] n_{i} Q_{i}}{1-R)}
\end{aligned}
$$

Case $4\left(L_{m} \geq X, L_{r} \geq Y\right)$. If $L_{m} \geq X$ and $L_{r} \geq Y$, both the manufacturer and the retailer will not earn interests income but need to pay opportunity costs, and the retailer's interests income and opportunity costs will be transferred into opportunity costs for the manufacturer. Consider the following.

(i) Opportunity cost $=C_{m} I_{\mathrm{mp}}(n Q-D X)^{2} / 2[1-Z(1-$ R) $] n_{i} Q_{i}$. (ii) Interest income $=C_{r} I_{\text {me }}(D X)^{2} / 2[1-Z(1-R)] n_{i} Q_{i}$.

(iii) Transfer opportunity cost $=C_{m} I_{\mathrm{mp}}(D Y)^{2} / 2 Q_{i}$.

(iv) Transfer interest income $=C_{r} I_{\mathrm{me}}\left(Q_{i}-D Y\right)^{2} / 2 Q_{i}$.

Thus, $\mathrm{TP}_{m 4}$ is given by

$\mathrm{TP}_{m 4}$

$=$ sales revenue - ordering cost - holding cost

- transportation cost - inspecting cost - repair cost

- scrap cost - opportunity cost + interest income

- transfer opportunity cost + transfer interest income

$$
\begin{gathered}
=D\left[C_{r}-\frac{C_{m}}{1-Z(1-R)}\right]-\frac{A_{m} D}{n_{i} Q_{i}} \\
-h_{m} D\left\{Q_{i}\left[\frac{n_{i}-1}{2 D}+\frac{\{1-2[1-Z(1-R)]\} n_{i}}{2 P[1-Z(1-R)]^{2}}+\frac{1}{P}\right]\right. \\
\left.\quad-\frac{t_{s} Z R n_{i}}{1-Z(1-R)}\right\} \\
-\frac{F_{m} D}{n_{i} Q_{i}}-\frac{D\left[W+C_{\mathrm{rm}} Z R+G Z(1-R)\right]}{1-Z(1-R)} \\
-\frac{C_{m} I_{\mathrm{mp}}(n Q-D X)^{2}}{2[1-Z(1-R)] n_{i} Q_{i}}+\frac{C_{r} I_{\mathrm{me}}(D X)^{2}}{2[1-Z(1-R)] n_{i} Q_{i}} \\
-\frac{C_{m} I_{\mathrm{mp}}(D Y)^{2}}{2 Q_{i}}+\frac{C_{r} I_{\mathrm{me}}\left(Q_{i}-D Y\right)^{2}}{2 Q_{i}} .
\end{gathered}
$$

3.3. The Retailer's Total Annual Profit. In each production run, the retailer's revenue includes sales revenue and interests income; the retailer's costs include ordering costs, holding costs, transportation costs, and opportunity costs. The relationship between the retailer's lead time $\left(L_{r}\right)$ and payment time $(Y)$ has been discussed before. The retailer may gain additional interests income or pay opportunity costs according to two different cases shown as follows.

Case $1\left(L_{r}<Y\right)$. If $L_{r}<Y$, the retailer will earn interest income. Consider the following.

(i) Sales revenue $=D\left(P_{r}-C_{r}\right)$.

(ii) Ordering cost $=A_{r} D / n_{i} Q_{i}$.

(iii) Holding cost $=h_{r} Q_{i} / 2$.

(iv) Transportation cost $=F_{r} D / Q_{i}$.

(v) Interest income $=P_{r} I_{\mathrm{re}}\left(D Y-Q_{i} / 2\right)$. 
Thus, $\mathrm{TP}_{r 1}$ is given by $\mathrm{TP}_{r 1}$

$=$ sales revenue - ordering cost - holding cost

- transportation cost + interest income

$=D\left(P_{r}-C_{r}\right)-\frac{A_{r} D}{n_{i} Q_{i}}-\frac{h_{r} Q_{i}}{2}-\frac{F_{r} D}{Q_{i}}+P_{r} I_{\mathrm{re}}\left(D Y-\frac{Q_{i}}{2}\right)$.

Case $2\left(L_{r} \geq Y\right)$. If $L_{r} \geq Y$, the retailer will not only earn interests income but also pay opportunity costs due to the rest of stocks. Consider the following.

(i) Opportunity cost $=C_{r} I_{\mathrm{rp}}\left(Q_{i}-D Y\right)^{2} / 2 Q_{i}$.

(ii) Interest income $=P_{r} I_{\mathrm{re}}(D Y)^{2} / 2 Q_{i}$.

Thus, $\mathrm{TP}_{r 2}$ is given by $\mathrm{TP}_{r 2}$

$=$ sales revenue - ordering cost - holding cost

- transportation cost - opportunity cost

+ interest income

$$
\begin{aligned}
= & D\left(P_{r}-C_{r}\right)-\frac{A_{r} D}{n_{i} Q_{i}}-\frac{h_{r} Q_{i}}{2}-\frac{F_{r} D}{Q_{i}}-\frac{C_{r} I_{\mathrm{rp}}\left(Q_{i}-D Y\right)^{2}}{2 Q_{i}} \\
& +\frac{P_{r} I_{\mathrm{re}}(D Y)^{2}}{2 Q_{i}} .
\end{aligned}
$$

3.4. The Expected Joint Total Annual Profit. According to different conditions, the expected joint total annual profit function, $\operatorname{EJTP}\left(Q_{i}, n_{i}\right)$, can be expressed as

$$
\operatorname{EJTP}_{i}\left(Q_{i}, n_{i}\right)
$$

$$
=\left\{\begin{aligned}
\operatorname{EJTP}_{1}\left(Q_{1}, n_{1}\right)= & \mathrm{TP}_{s 1}+\mathrm{TP}_{m 1}+\mathrm{TP}_{r 1} \\
& \text { if } L_{m}<X, L_{r}<Y \\
\operatorname{EJTP}_{2}\left(Q_{2}, n_{2}\right)= & \mathrm{TP}_{s 1}+\mathrm{TP}_{m 2}+\mathrm{TP}_{r 2} \\
& \text { if } L_{m}<X, L_{r} \geq Y \\
\operatorname{EJTP}_{3}\left(Q_{3}, n_{3}\right)= & \mathrm{TP}_{s 2}+\mathrm{TP}_{m 3}+\mathrm{TP}_{r 1} \\
& \text { if } L_{m} \geq X, L_{r}<Y \\
\operatorname{EJTP}_{4}\left(Q_{4}, n_{4}\right)= & \mathrm{TP}_{s 2}+\mathrm{TP}_{m 4}+\mathrm{TP}_{r 2} \\
& \text { if } L_{m} \geq X, L_{r} \geq Y,
\end{aligned}\right.
$$

where

$$
\begin{aligned}
& \operatorname{EJTP}_{1}\left(Q_{1}, n_{1}\right) \\
& =D\left[P_{r}-\frac{C_{s}+W+C_{\mathrm{rm}} Z R+G Z(1-R)}{1-Z(1-R)}\right] \\
& -h_{m} D\left\{Q_{1}\left[\frac{n_{1}-1}{2 D}+\frac{\{1-2[1-Z(1-R)]\} n_{1}}{2 P[1-Z(1-R)]^{2}}+\frac{1}{P}\right]\right. \\
& \left.\quad-\frac{t_{s} Z R n_{1}}{1-Z(1-R)}\right\}-\frac{h_{r} Q_{1}}{2}-\frac{h_{s} D n_{1} Q_{1}}{2 P[1-Z(1-R)]^{2}}
\end{aligned}
$$

$$
\begin{aligned}
& -\frac{D\left(A_{s}+A_{m}+F_{m}+A_{r}+F_{r} n_{1}\right)}{n_{1} Q_{1}} \\
& +\frac{\left(C_{r} I_{\mathrm{me}}-C_{s} I_{\mathrm{sp}}\right)\left(2 D X-n_{1} Q_{1}\right)}{2[1-Z(1-R)]} \\
& +\left(P_{r} I_{\mathrm{re}}-C_{m} I_{\mathrm{mp}}\right)\left(D Y-\frac{Q_{1}}{2}\right),
\end{aligned}
$$$$
\operatorname{EJTP}_{2}\left(Q_{2}, n_{2}\right)
$$$$
=D\left[P_{r}-\frac{C_{s}+W+C_{\mathrm{rm}} Z R+G Z(1-R)}{1-Z(1-R)}\right]
$$$$
-h_{m} D\left\{Q_{2}\left[\frac{n_{2}-1}{2 D}+\frac{\{1-2[1-Z(1-R)]\} n_{2}}{2 P[1-Z(1-R)]^{2}}+\frac{1}{P}\right]\right.
$$$$
\left.-\frac{t_{s} Z R n_{2}}{1-Z(1-R)}\right\}-\frac{h_{r} Q_{2}}{2}-\frac{h_{s} D n_{2} Q_{2}}{2 P[1-Z(1-R)]^{2}}
$$$$
-\frac{D\left(A_{s}+A_{m}+F_{m}+A_{r}+F_{r} n_{2}\right)}{n_{2} Q_{2}}
$$$$
+\frac{\left(C_{r} I_{\mathrm{me}}-C_{s} I_{\mathrm{sp}}\right)\left(2 D X-n_{2} \mathrm{Q}_{2}\right)}{2[1-Z(1-R)]}
$$$$
+\frac{\left(C_{r} I_{\mathrm{me}}-C_{r} I_{\mathrm{rp}}\right)\left(Q_{2}-D Y\right)^{2}}{2 Q_{2}}+\frac{\left(P_{r} I_{\mathrm{re}}-C_{m} I_{\mathrm{mp}}\right)(D Y)^{2}}{2 Q_{2}} \text {, }
$$

$\operatorname{EJTP}_{3}\left(Q_{3}, n_{3}\right)$

$$
\begin{aligned}
= & D\left[P_{r}-\frac{C_{s}+W+C_{\mathrm{rm}} Z R+G Z(1-R)}{1-Z(1-R)}\right] \\
- & h_{m} D\left\{Q_{3}\left[\frac{n_{3}-1}{2 D}+\frac{\{1-2[1-Z(1-R)]\} n_{3}}{2 P[1-Z(1-R)]^{2}}+\frac{1}{P}\right]\right. \\
& \left.-\frac{t_{s} Z R n_{3}}{1-Z(1-R)}\right\}-\frac{h_{r} Q_{3}}{2}-\frac{h_{s} D n_{3} Q_{3}}{2 P[1-Z(1-R)]^{2}} \\
+ & \frac{D\left(A_{s}+A_{m}+F_{m}+A_{r}+F_{r} n_{3}\right)}{n_{3} Q_{3}} \\
+ & \frac{\left(C_{r} I_{\mathrm{me}}-C_{s} I_{\mathrm{sp}}\right)(D X)^{2}}{2[1-Z(1-R)] n_{3} Q_{3}} \\
+ & \frac{\left(C_{m} I_{\mathrm{se}}-C_{m} I_{\mathrm{mp}}\right)\left(n_{3} Q_{3}-D X\right)^{2}}{2[1-Z(1-R)] n_{3} Q_{3}} \\
+ & \frac{\left.\left(C_{m} I_{\mathrm{se}}-C_{m} I_{\mathrm{mp}}\right)\left(n_{3} Q_{3}-D X\right)_{\mathrm{re}}-C_{m} I_{\mathrm{mp}}\right)\left(D Y-\frac{Q_{3}}{2[1-Z(1-R)] n_{3} Q_{3}}\right.}{2} \\
+ & \\
+ &
\end{aligned}
$$

$\operatorname{EJTP}_{4}\left(Q_{4}, n_{4}\right)$

$$
=D\left[P_{r}-\frac{C_{s}+W+C_{\mathrm{rm}} Z R+G Z(1-R)}{1-Z(1-R)}\right]
$$




$$
\begin{aligned}
& -h_{m} D\left\{Q_{4}\left[\frac{n_{4}-1}{2 D}+\frac{\{1-2[1-Z(1-R)]\} n_{4}}{2 P[1-Z(1-R)]^{2}}+\frac{1}{P}\right]\right. \\
& \left.\quad-\frac{t_{s} Z R n_{4}}{1-Z(1-R)}\right\}-\frac{h_{r} Q_{4}}{2}-\frac{h_{s} D n_{4} Q_{4}}{2 P[1-Z(1-R)]^{2}} \\
& -\frac{D\left(A_{s}+A_{m}+F_{m}+A_{r}+F_{r} n_{4}\right)}{n_{4} Q_{4}} \\
& +\frac{\left(C_{r} I_{\mathrm{me}}-C_{s} I_{\mathrm{sp}}\right)(D X)^{2}}{2[1-Z(1-R)] n_{4} Q_{4}} \\
& +\frac{\left(C_{m} I_{\mathrm{se}}-C_{m} I_{\mathrm{mp}}\right)\left(n_{4} Q_{4}-D X\right)^{2}}{2[1-Z(1-R)] n_{4} Q_{4}} \\
& +\frac{\left(C_{r} I_{\mathrm{me}}-C_{r} I_{\mathrm{rp}}\right)\left(Q_{4}-D Y\right)^{2}}{2 Q_{4}}+\frac{\left(P_{r} I_{\mathrm{re}}-C_{m} I_{\mathrm{mp}}\right)(D Y)^{2}}{2 Q_{4}} .
\end{aligned}
$$

\section{Solution Procedure}

4.1. Determination of the Optimal Delivery Quantity $Q_{i}$ for Any Given $n_{i}$. We would like to find the maximum value of the expected total profit $\operatorname{EJTP}\left(Q_{i}, n_{i}\right)$. For any $n_{i}$, we will take the first and second partial derivations of $\operatorname{EJTP}\left(Q_{i}, n_{i}\right)$ with respect to $Q_{i}$. We have

$$
\begin{aligned}
\frac{\partial \mathrm{EJTP}_{1}\left(Q_{1}, n_{1}\right)}{\partial Q_{1}} & \frac{D\left(A_{s}+A_{m}+F_{m}+A_{r}+F_{r} n_{1}\right)}{n_{1} Q_{1}^{2}} \\
= & -h_{m} D\left\{\frac{n_{1}-1}{2 D}+\frac{\{1-2[1-Z(1-R)]\} n_{1}}{2 P[1-Z(1-R)]^{2}}+\frac{1}{P}\right\} \\
& -\frac{h_{r}}{2}-\frac{h_{s} D n_{1}}{2 P[1-Z(1-R)]^{2}}-\frac{\left(C_{r} I_{\mathrm{me}}-C_{s} I_{\mathrm{sp}}\right) n_{1}}{2[1-Z(1-R)]} \\
& -\frac{\left(P_{r} I_{\mathrm{re}}-C_{m} I_{\mathrm{mp}}\right)}{2},
\end{aligned}
$$

$$
\begin{aligned}
& \frac{\partial \mathrm{EJTP}_{2}\left(Q_{2}, n_{2}\right)}{\partial Q_{2}} \\
& =\frac{D\left(A_{s}+A_{m}+F_{m}+A_{r}+F_{r} n_{2}\right)}{n_{2} Q_{2}^{2}} \\
& \quad-h_{m} D\left\{\frac{n_{2}-1}{2 D}+\frac{\{1-2[1-Z(1-R)]\} n_{2}}{2 P[1-Z(1-R)]^{2}}+\frac{1}{P}\right\} \\
& \quad-\frac{h_{r}}{2}-\frac{h_{s} D n_{2}}{2 P[1-Z(1-R)]^{2}}-\frac{\left(C_{r} I_{\mathrm{me}}-C_{s} I_{\mathrm{sp}}\right) n_{2}}{2[1-Z(1-R)]}
\end{aligned}
$$

$$
\begin{aligned}
& -\frac{\left(P_{r} I_{\mathrm{re}}-C_{m} I_{\mathrm{mp}}\right)(D Y)^{2}}{2 Q_{2}^{2}} \\
& +\frac{\left(C_{r} I_{\mathrm{me}}-C_{r} I_{\mathrm{rp}}\right)\left[Q_{2}^{2}-(D Y)^{2}\right]}{2 Q_{2}^{2}}
\end{aligned}
$$

$$
\begin{aligned}
& \frac{\partial \mathrm{EJTP}_{3}\left(Q_{3}, n_{3}\right)}{\partial Q_{3}} \\
& =\frac{D\left(A_{s}+A_{m}+F_{m}+A_{r}+F_{r} n_{3}\right)}{n_{3} Q_{3}^{2}} \\
& -h_{m} D\left\{\frac{n_{3}-1}{2 D}+\frac{\{1-2[1-Z(1-R)]\} n_{3}}{2 P[1-Z(1-R)]^{2}}+\frac{1}{P}\right\} \\
& -\frac{h_{r}}{2}-\frac{h_{s} D n_{3}}{2 P[1-Z(1-R)]^{2}} \\
& -\frac{\left(C_{r} I_{\mathrm{me}}-C_{s} I_{\mathrm{sp}}\right)(D X)^{2}}{2[1-Z(1-R)] n_{3} Q_{3}^{2}} \\
& +\frac{\left(C_{m} I_{\mathrm{se}}-C_{m} I_{\mathrm{mp}}\right)\left[\left(n_{3} Q_{3}\right)^{2}-(D X)^{2}\right]}{2[1-Z(1-R)] n_{3} Q_{3}^{2}} \\
& -\frac{\left(P_{r} I_{\mathrm{re}}-C_{m} I_{\mathrm{mp}}\right)}{2} \\
& \frac{\partial \operatorname{EJTP}_{4}\left(Q_{4}, n_{4}\right)}{\partial Q_{4}} \\
& =\frac{D\left(A_{s}+A_{m}+F_{m}+A_{r}+F_{r} n_{4}\right)}{n_{4} Q_{4}^{2}} \\
& -h_{m} D\left\{\frac{n_{4}-1}{2 D}+\frac{\{1-2[1-Z(1-R)]\} n_{4}}{2 P[1-Z(1-R)]^{2}}+\frac{1}{P}\right\} \\
& -\frac{h_{r}}{2}-\frac{h_{s} D n_{4}}{2 P[1-Z(1-R)]^{2}}-\frac{\left(C_{r} I_{\mathrm{me}}-C_{s} I_{\mathrm{sp}}\right)(D X)^{2}}{2[1-Z(1-R)] n_{4} Q_{4}^{2}} \\
& -\frac{\left(P_{r} I_{\mathrm{re}}-C_{m} I_{\mathrm{mp}}\right)(D Y)^{2}}{2 Q_{4}^{2}} \\
& +\frac{\left(C_{m} I_{\mathrm{se}}-C_{m} I_{\mathrm{mp}}\right)\left[\left(n_{4} \mathrm{Q}_{4}\right)^{2}-(D X)^{2}\right]}{2[1-Z(1-R)] n_{4} Q_{4}^{2}} \\
& +\frac{\left(C_{r} I_{\mathrm{me}}-C_{r} I_{\mathrm{rp}}\right)\left[Q_{4}^{2}-(D Y)^{2}\right]}{2 Q_{4}^{2}}
\end{aligned}
$$




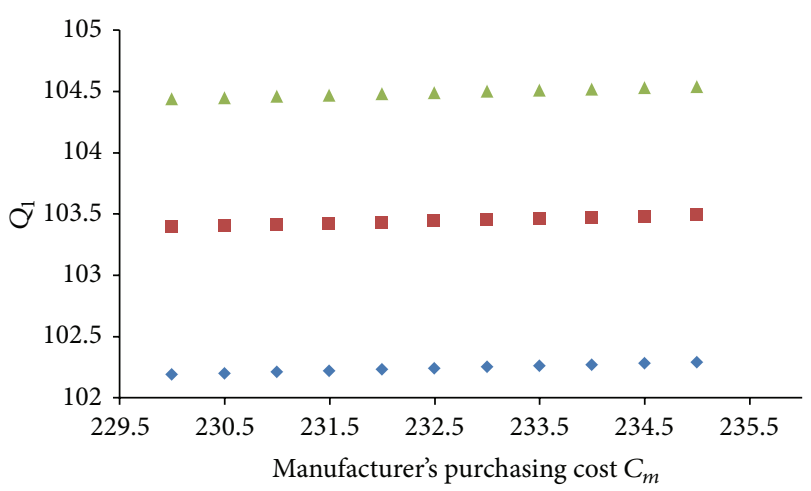

(a) The value of $Q_{1}$ by changing $C_{m}$ under different $P$

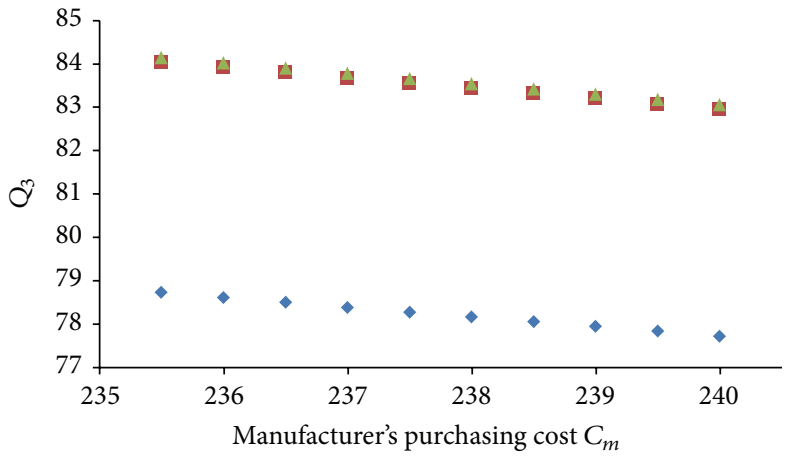

- $P=1100$

- $P=1200$

$\triangle P=1300$

(c) The value of $Q_{3}$ by changing $C_{m}$ under different $P$

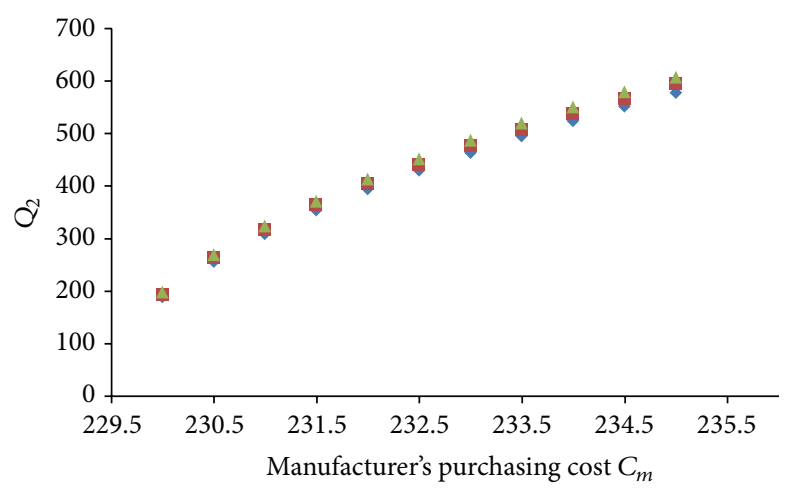

(b) The value of $Q_{2}$ by changing $C_{m}$ under different $P$

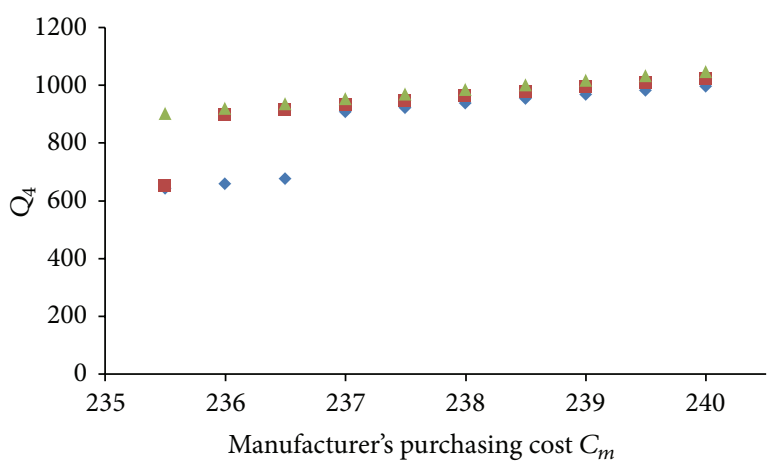

- $P=1100$

- $P=1200$

A $P=1300$

(d) The value of $Q_{4}$ by changing $C_{m}$ under different $P$

Figure 5: The value of delivery quantity by changing $C_{m}$ in $Q_{i}$, for $i=1,2,3,4$.

$$
\begin{aligned}
& \frac{\partial^{2} \operatorname{EJTP}_{1}\left(Q_{1}, n_{1}\right)}{\partial Q_{1}^{2}} \\
& \quad=-\frac{2 D\left(A_{s}+A_{m}+F_{m}+A_{r}+F_{r} n_{1}\right)}{n_{1} Q_{1}^{3}}<0,
\end{aligned}
$$

$$
\begin{aligned}
& \frac{\partial^{2} \mathrm{EJTP}_{2}\left(Q_{2}, n_{2}\right)}{\partial Q_{2}^{2}} \\
& =-\frac{2 D\left(A_{s}+A_{m}+F_{m}+A_{r}+F_{r} n_{2}\right)}{n_{2} Q_{2}^{3}} \\
& \quad+\frac{\left(C_{r} I_{\mathrm{me}}-C_{r} I_{\mathrm{rp}}\right)(D Y)^{2}}{Q_{2}^{3}}+\frac{\left(P_{r} I_{\mathrm{re}}-C_{m} I_{\mathrm{mp}}\right)(D Y)^{2}}{Q_{2}^{3}}
\end{aligned}
$$$$
<0 \text {, }
$$

$$
\begin{aligned}
& \frac{\partial^{2} \operatorname{EJTP}_{3}\left(Q_{3}, n_{3}\right)}{\partial Q_{3}^{2}} \\
& =-\frac{2 D\left(A_{s}+A_{m}+F_{m}+A_{r}+F_{r} n_{3}\right)}{n_{3} Q_{3}^{3}}
\end{aligned}
$$

$$
\begin{aligned}
\frac{\partial^{2} \mathrm{EJTP}_{4}\left(Q_{4}, n_{4}\right)}{\partial Q_{4}^{2}} & \\
=- & \frac{2 D\left(A_{s}+A_{m}+F_{m}+A_{r}+F_{r} n_{4}\right)}{n_{4} Q_{4}^{3}} \\
+ & \frac{\left(C_{r} I_{\mathrm{me}}-C_{s} I_{\mathrm{sp}}\right)(D X)^{2}}{[1-Z(1-R)] n_{4} Q_{4}^{3}}+\frac{\left(C_{m} I_{\mathrm{se}}-C_{m} I_{\mathrm{mp}}\right)(D X)^{2}}{[1-Z(1-R)] n_{4} Q_{4}^{3}} \\
+ & \frac{\left(C_{r} I_{\mathrm{me}}-C_{r} I_{\mathrm{rp}}\right)(D Y)^{2}}{Q_{4}^{3}}+\frac{\left(P_{r} I_{\mathrm{re}}-C_{m} I_{\mathrm{mp}}\right)(D Y)^{2}}{Q_{4}^{3}}
\end{aligned}
$$

$<0$.

Because (16), (17), (18), and (19) $<0$, therefore $\operatorname{EJTP}\left(Q_{i}, n_{i}\right)$ is concave function in $Q_{i}$ for fixed $n_{i}$. We can find a unique value of $Q_{i}$ that maximize $\operatorname{EJTP}\left(Q_{i}, n_{i}\right)$. Let 


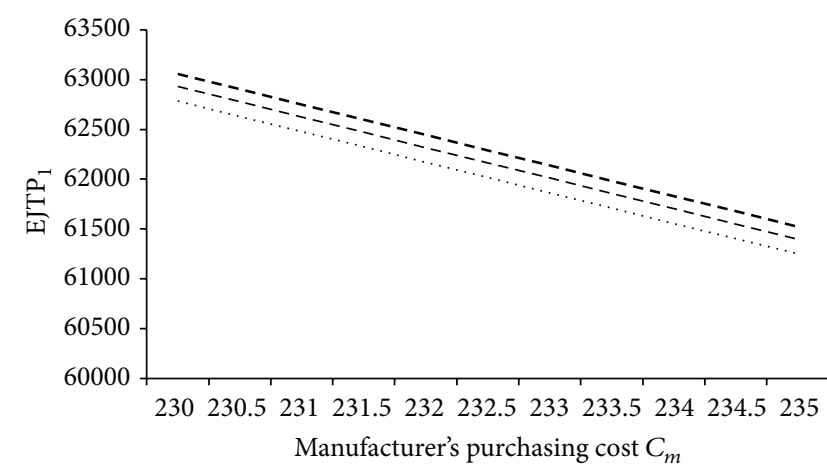

(a) The value of $\mathrm{EJTP}_{1}$ by changing $C_{m}$ under different $P$

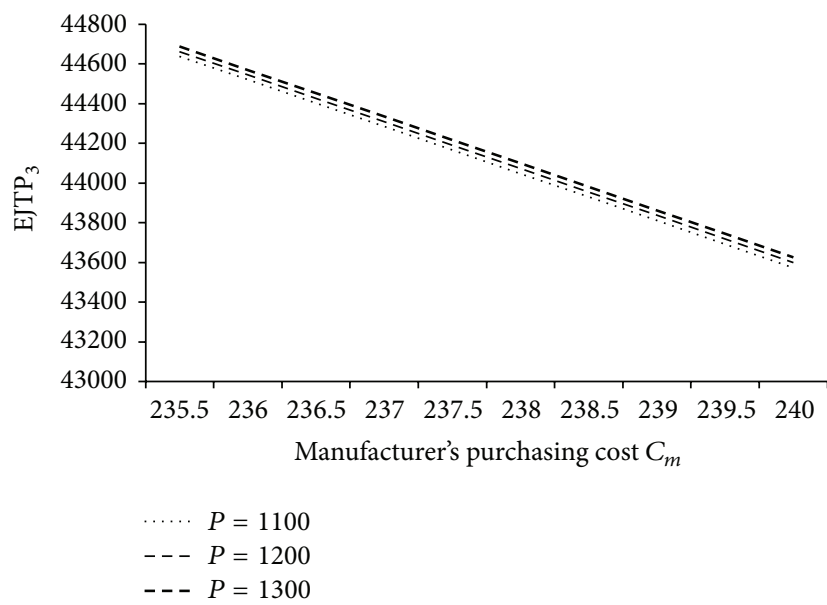

(c) The value of $\mathrm{EJTP}_{3}$ by changing $C_{m}$ under different $P$

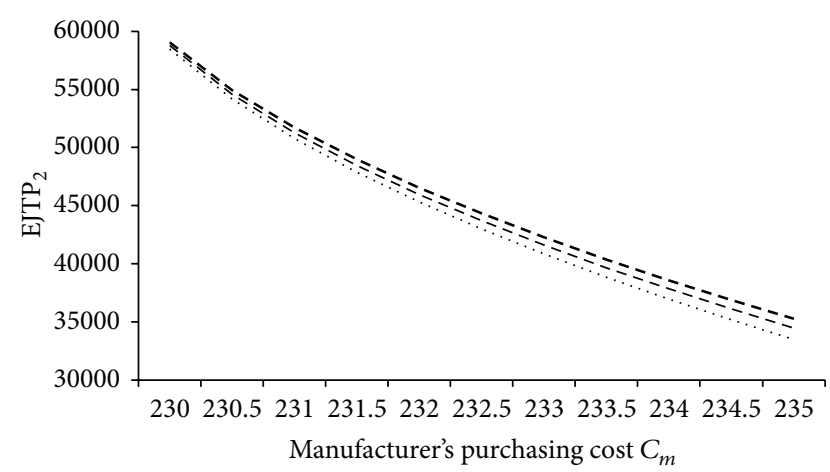

(b) The value of $\mathrm{EJTP}_{2}$ by changing $C_{m}$ under different $P$

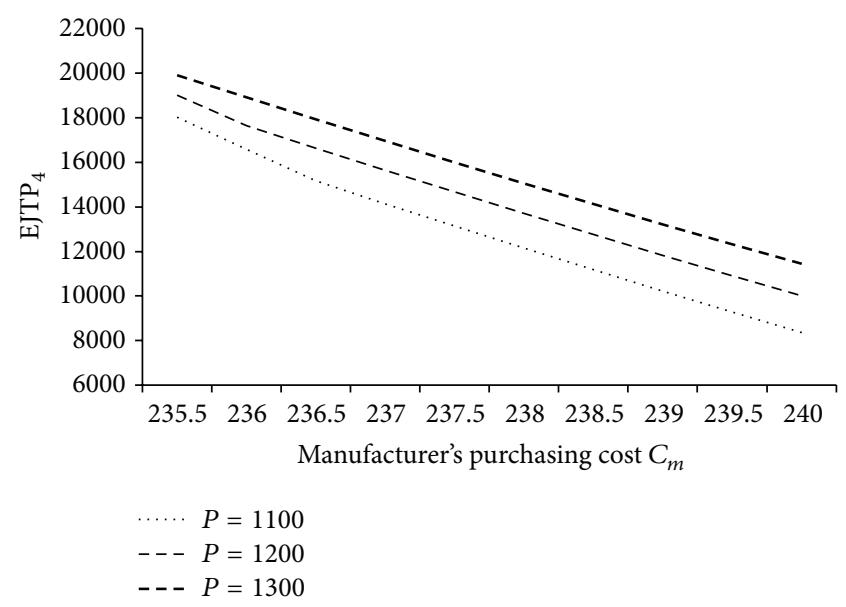

(d) The value of $\mathrm{EJTP}_{4}$ by changing $C_{m}$ under different $P$

FIgure 6: The value of profit by changing $C_{m}$ in $\operatorname{EJTP}_{i}$, for $i=1,2,3,4$.

$\operatorname{DEJTP}_{i}\left(Q_{i}, n_{i}\right) / \partial Q_{i}=0$ in (16), (17), (18), and (19), so we can get that $Q_{i}$ are as follows.

The original equations are too long, so in order to shorten them, we let $[1-Z(1-R)]=U ;\left(C_{r} I_{\mathrm{me}}-C_{s} I_{\mathrm{sp}}\right)=M ;\left(P_{r} I_{\mathrm{re}}-\right.$ $\left.C_{m} I_{\mathrm{mp}}\right)=W ;\left(C_{r} I_{\mathrm{me}}-C_{r} I_{\mathrm{rp}}\right)=B ;\left(C_{m} I_{\mathrm{se}}-C_{m} I_{\mathrm{mp}}\right)=E$. Then we substitute them into the original equations

$$
\begin{aligned}
& Q_{1}^{*}=(\left(2 D P U^{2}\left(A_{s}+A_{m}+F_{m}+A_{r}+F_{r} n_{1}\right)\right) \\
& \times\left(n _ { 2 } \left\{P U\left[U\left(h_{m}\left(n_{1}-1\right)+h_{r}+W\right)+M n_{1}\right]\right.\right. \\
&\left.\left.\left.+D\left[n_{1}\left(h_{s}+h_{m}(1-2 U)\right)+2 h_{m} U^{2}\right]\right\}\right)^{-1}\right)^{1 / 2}
\end{aligned}
$$

$$
\begin{aligned}
& Q_{2}^{*}=\left(\left(P U^{2}[\right.\right. 2 D\left(A_{s}+A_{m}+F_{m}+A_{r}+F_{r} n_{2}\right) \\
&\left.\left.-n_{2}(B+W)(D Y)^{2}\right]\right) \\
& \times\left(n _ { 2 } \left\{P U\left[U\left(h_{m}\left(n_{2}-1\right)+h_{r}-B\right)+M n_{2}\right]\right.\right. \\
&\left.\left.\left.+D\left[n_{2}\left(h_{s}+h_{m}(1-2 U)\right)+2 h_{m} U^{2}\right]\right\}\right)^{-1}\right)^{1 / 2}
\end{aligned}
$$

$$
\begin{aligned}
& Q_{3}^{*}=( P U\left[2 D U\left(A_{s}+A_{m}+F_{m}+A_{r}+F_{r} n_{3}\right)\right. \\
&\left.\left.-(M+E)(D X)^{2}\right]\right) \times\left(n _ { 3 } \left\{P U\left[U\left(h_{m}\left(n_{3}-1\right)+h_{r}+W\right)-E\right]\right.\right. \\
&\left.\left.\left.+D\left[n_{3}\left(h_{s}+h_{m}(1-2 U)\right)+2 h_{m} U^{2}\right]\right\}\right)^{-1}\right)^{1 / 2}, \\
& Q_{4}^{*}=\left(\left(P U \left[2 D U\left(A_{s}+A_{m}+F_{m}+A_{r}+F_{r} n_{4}\right)\right.\right.\right. \\
&\left.\left.-(M+E)(D X)^{2}-U n_{4}(B+W)(D Y)^{2}\right]\right) \\
& \times\left(n _ { 4 } \left\{P U\left[U\left(h_{m}\left(n_{4}-1\right)+h_{r}-B\right)-E\right]\right.\right. \\
&\left.\left.\left.+D\left[n_{4}\left(h_{s}+h_{m}(1-2 U)\right)+2 h_{m} U^{2}\right]\right\}\right)^{-1}\right)^{1 / 2} .
\end{aligned}
$$

Algorithm. To summarize the above arguments, we established the algorithm to obtain the optimal values of $\operatorname{EJTP}\left(n_{i}, Q_{i}\right)$.

Equation (10) shows the situations of each case; obviously, each case is mutual exclusive. In other words, before we start 


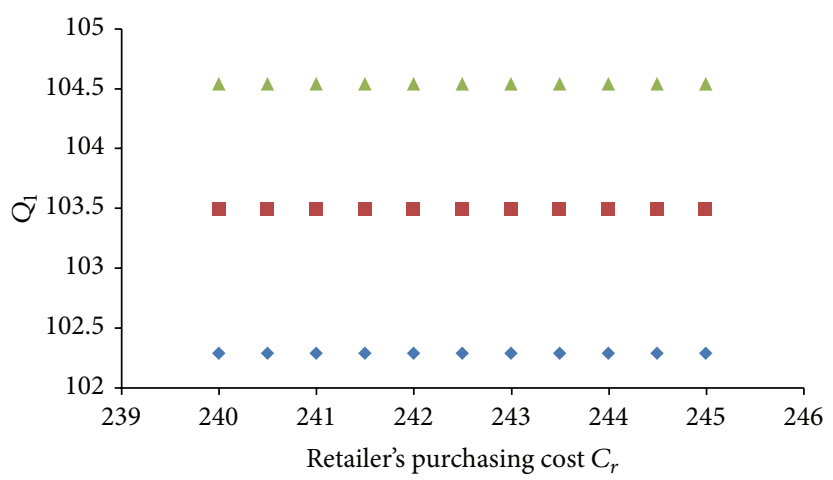

(a) The value of $Q_{1}$ by changing $C_{r}$ under different $P$

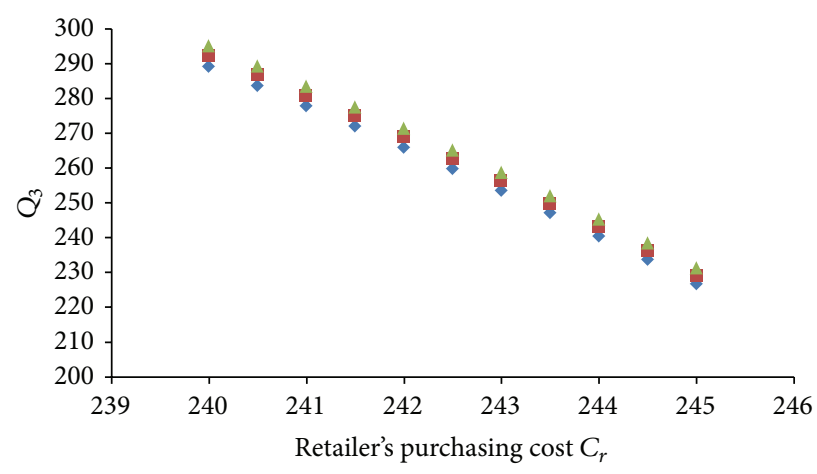

- $P=1100$

- $P=1200$

$\triangle P=1300$

(c) The value of $Q_{3}$ by changing $C_{r}$ under different $P$

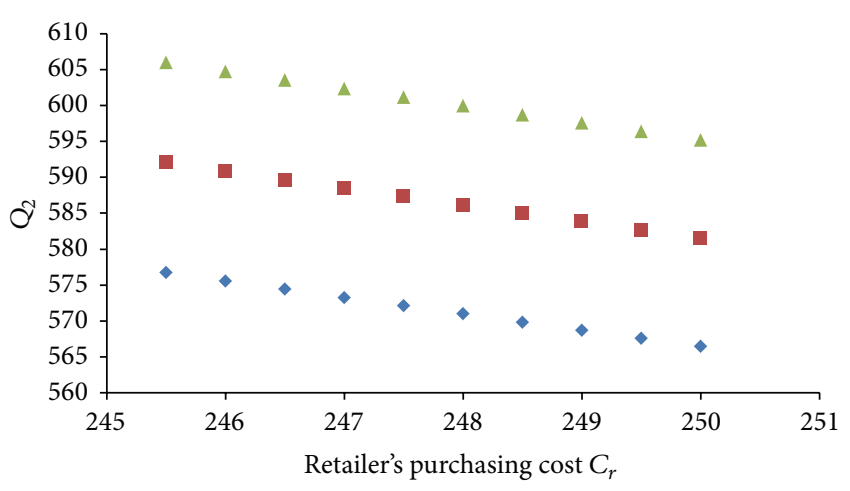

(b) The value of $Q_{2}$ by changing $C_{r}$ under different $P$

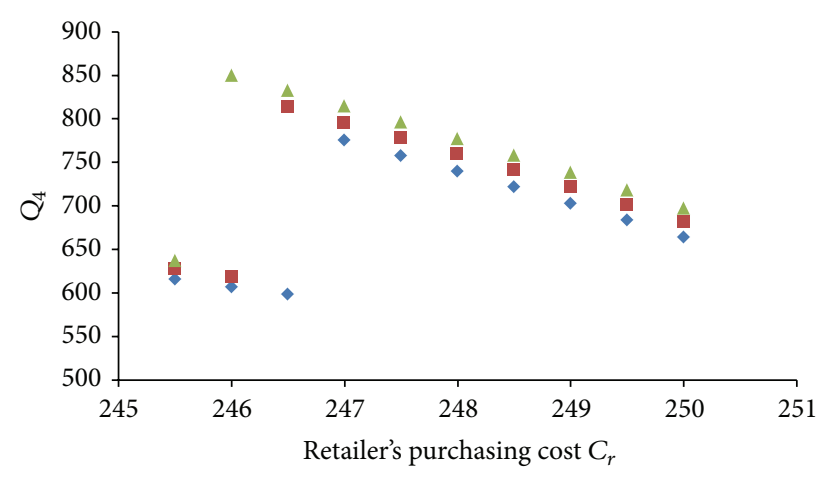

- $P=1100$

- $P=1200$

$\triangle P=1300$

(d) The value of $\mathrm{Q}_{4}$ by changing $C_{r}$ under different $P$

FIgURE 7: The value of delivery quantity by changing $C_{r}$ in $Q_{i}$, for $i=1,2,3,4$.

to find the optimal solutions, we have to recognize which equations should be used first.

Step 1. Examine the relationship of $L_{m}, X$ and $L_{r}, Y$ to use corresponding equations.

Step 2. Let $n_{i}=1$ and substitute into (20), (21), (22), or (23) to find $Q_{1}, Q_{2}, Q_{3}$, or $Q_{4}$.

Step 3. Find $\mathrm{EJTP}_{i}$ by substituting $n_{i}, Q_{i}$, and different production rate $(P)$.

Step 4. Let $n=n_{i}+1$ and repeat Step 2 to Step 3 until $\operatorname{EJTP}_{i\left(n_{i}\right)}>\operatorname{EJTP}_{i\left(n_{i}+1\right)}$.

\section{Numerical Example}

In Section 5, we will observe the variation of $Q_{i}, n_{i}$, and $\mathrm{EJTP}_{i}$ by changing $C_{m}$ and $C_{r}$ separately under different production rate or defective rate. We consider an inventory system with the following data.

Consider $D=1000$ unit/year, $C_{s}=200$ per unit, $A_{s}=80$ per order, $h_{s}=20$ per unit, $I_{\text {sp }}=0.025$ per year, $I_{\text {se }}=0.0254$ per year, $C_{m}=235$ per unit, $A_{m}=100$ per order, $h_{m}=23$ per unit, $W=5$ per unit, $C_{\mathrm{rm}}=10$ per unit, $G=10$ per unit, $F_{m}=100$ per time, $Z=0.1, R=0.9, t_{s}=0.0055$ year, $I_{\mathrm{mp}}=0.0256$ per year, $I_{\mathrm{me}}=0.02$ per year, $C_{r}=245$ per unit, $A_{r}=120$ per order, $h_{r}=25$ per unit, $F_{r}=150$ per time, $P_{r}=280$ per unit, $I_{\mathrm{rp}}=0.02$ per year, and $I_{\mathrm{re}}=0.021$ per year.

5.1. The Variation under Different P. In Section 5.1, we supposed that the maximum of the production rate is 1300 . The manufacturer can change the production rate under any condition; furthermore, the extra payment by changing the rate is ignored. Let us observe the value of delivery quantity and profit with $P=1100, P=1200$, and $P=1300$ by changing the manufacturer's purchasing costs, and we set the function of $L_{m}$ and $X$ is $X=3000 / C_{m}$, or changing the retailer's purchasing costs, and we set the function of $L_{r}$ and $Y$ is $Y=3000 / C_{r}$.

5.1.1. The Permissible Period $X$ and EJTP. We have changed $C_{m}$ by 0.5 per unit. In order to find out which condition is more beneficial to the proposed inventory model, we formed the details shown in Table 1 and the solution results are illustrated in Figures 5 and 6.

We have discussed that if the payment time is longer than the lead time, it will bring additional interests income 
TABLE 1: The value of profit in different condition by changing $C_{m}$.

\begin{tabular}{|c|c|c|c|}
\hline & $P=1100$ & $P=1200$ & $P=1300$ \\
\hline$C_{m}$ & $230.0 \sim 235.0$ & $230.0 \sim 235.0$ & $230.0 \sim 235.0$ \\
\hline$n_{1}$ & 2 & 2 & 2 \\
\hline$Q_{1}$ & $102.19 \sim 102.29$ & $103.39 \sim 103.49$ & $104.44 \sim 104.54$ \\
\hline $\mathrm{EJTP}_{1}$ & $62782.89 \sim 61249.25$ & $62930.18 \sim 61396.67$ & ${ }^{*} 63056.73 \sim 61523.33$ \\
\hline$C_{m}$ & $230.0 \sim 235.0$ & $230.0 \sim 235.0$ & $230.0 \sim 235.0$ \\
\hline$n_{2}$ & 1 & 1 & 1 \\
\hline$Q_{2}$ & $189.02 \sim 577.86$ & $194.04 \sim 593.21$ & $198.62 \sim 607.21$ \\
\hline $\mathrm{EJTP}_{2}$ & $58465.23 \sim 33503.15$ & 58779.07 34462.59 & $59051.28 \sim 35294.77$ \\
\hline$C_{m}$ & $235.5 \sim 240$ & $235.5 \sim 240$ & $235.5 \sim 240$ \\
\hline$n_{3}$ & 14 & 13 & 13 \\
\hline$Q_{3}$ & 78.73 77.72 & $84.04 \sim 82.95$ & $84.15 \sim 83.06$ \\
\hline $\mathrm{EJTP}_{3}$ & $44637.85 \sim 43572.97$ & $44660.66 \sim 43599.22$ & $44686.91 \sim 43625.13$ \\
\hline$C_{m}$ & $235.5 \sim 240$ & $235.5 \sim 240$ & $235.5 \sim 240$ \\
\hline \multirow{2}{*}{$n_{4}$} & $2\left(C_{m}=235.5 \sim 236.5\right)$ & $2\left(C_{m}=235.5\right)$ & \multirow{2}{*}{1} \\
\hline & $1\left(C_{m}=235.5 \sim 236.5\right)$ & $1\left(C_{m}=236.0 \sim 236.5\right)$ & \\
\hline \multirow{2}{*}{$Q_{4}$} & $641.72 \sim 675.19\left(C_{m}=235.5 \sim 236.5\right)$ & $653.70\left(C_{m}=235.5\right)$ & \multirow{2}{*}{$901.78 \sim 1046.84$} \\
\hline & $906.62 \sim 996.36\left(C_{m}=237.0 \sim 240.0\right)$ & $897.88 \sim 1022.77\left(C_{m}=235.5 \sim 236.5\right)$ & \\
\hline $\mathrm{EJTP}_{4}$ & $18007.04 \sim 8353.20$ & $19000.21 \sim 10007.45$ & $19908.57 \sim 11442.18$ \\
\hline
\end{tabular}

${ }^{*}$ Optimal solution of $\mathrm{EJTP}_{i}$.

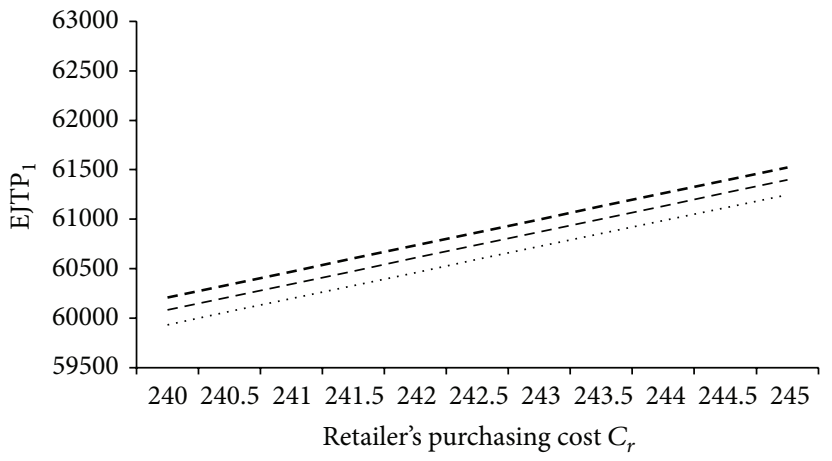

(a) The value of $\mathrm{EJTP}_{1}$ by changing $C_{r}$ under different $P$

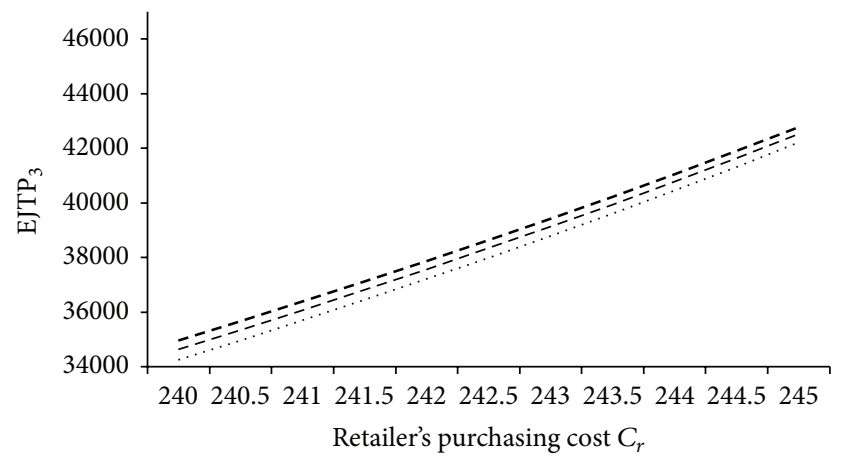

… $P=1100$

$---P=1200$

-- $P=1300$

(c) The value of $\mathrm{EJTP}_{3}$ by changing $C_{r}$ under different $P$

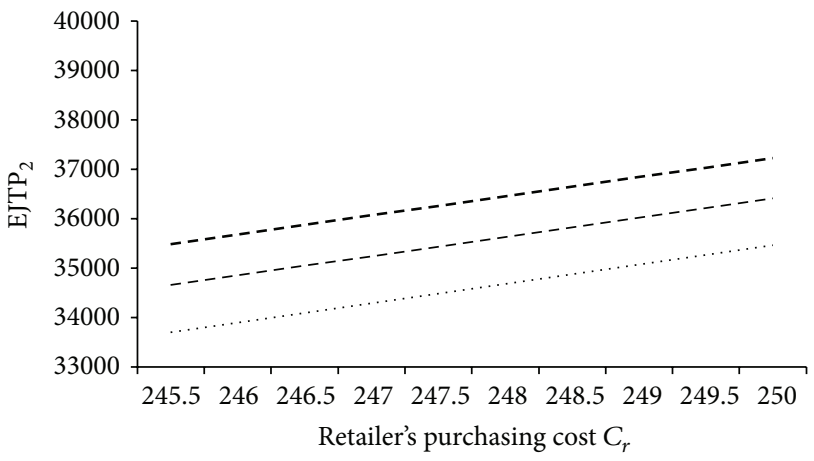

(b) The value of $\mathrm{EJTP}_{2}$ by changing $C_{r}$ under different $P$

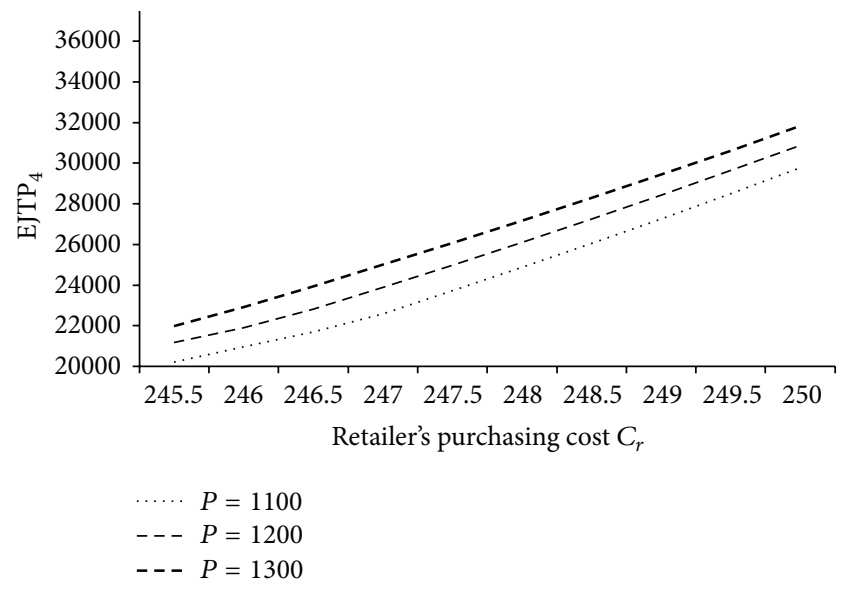

(d) The value of $\mathrm{EJTP}_{4}$ by changing $C_{r}$ under different $P$

FIgURE 8: The value of profit by changing $C_{r}$ in $\mathrm{EJTP}_{i}$, for $i=1,2,3,4$. 


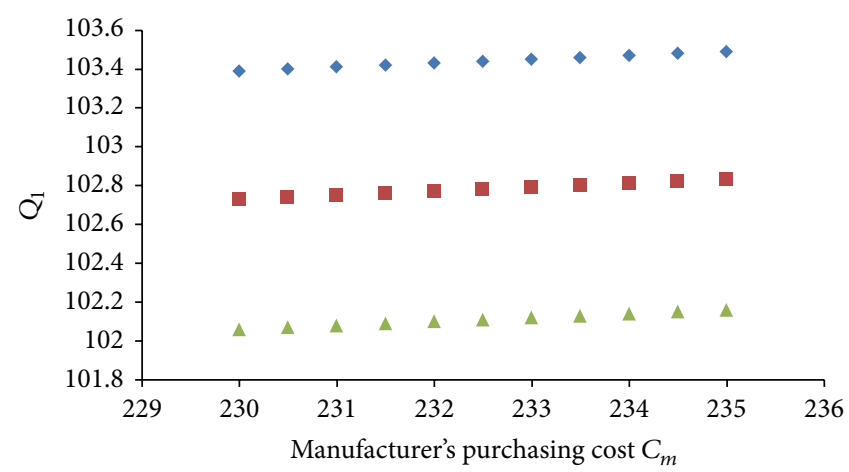

(a) The value of $Q_{1}$ by changing $C_{m}$ under different $Z$

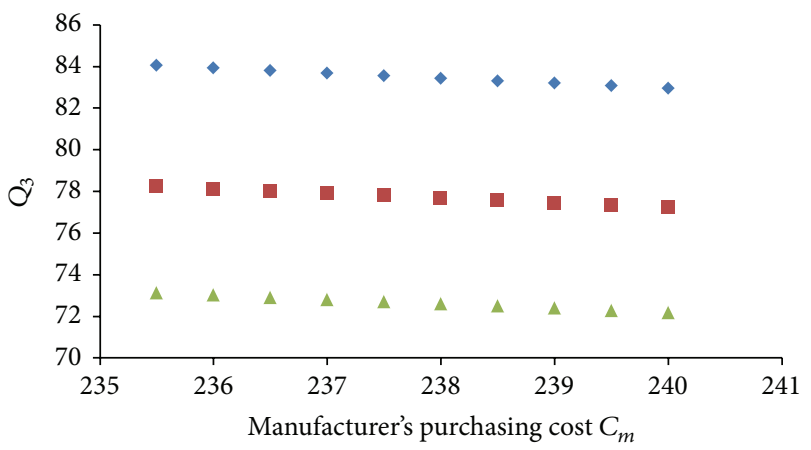

- $Z=0.1$

- $Z=0.2$

$\triangle Z=0.3$

(c) The value of $Q_{3}$ by changing $C_{m}$ under different $Z$

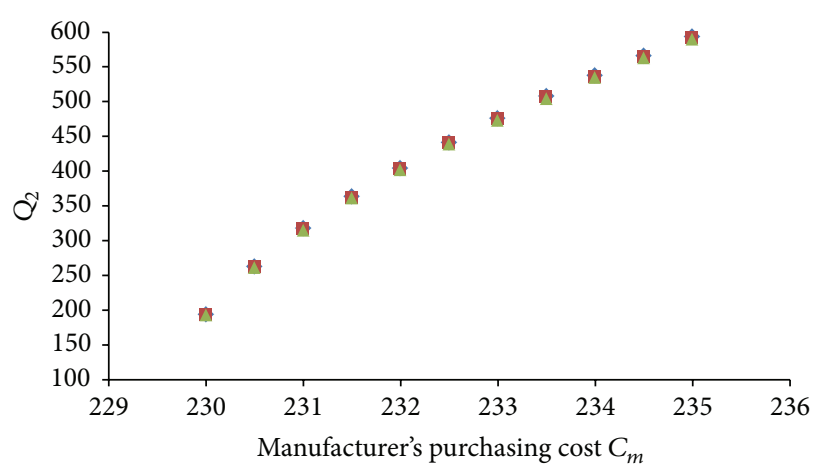

(b) The value of $Q_{2}$ by changing $C_{m}$ under different $Z$

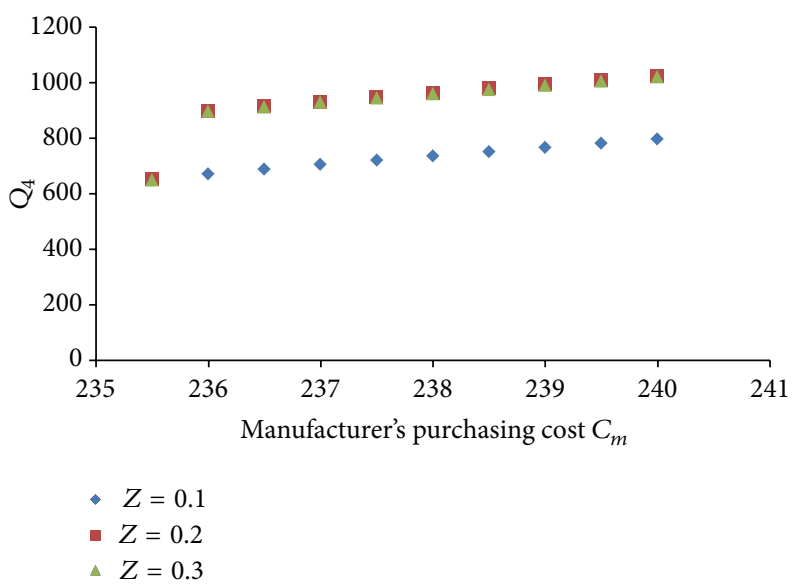

(d) The value of $Q_{4}$ by changing $C_{m}$ under different $Z$

FIGURE 9: The value of delivery quantity by changing $C_{m}$ in $Q_{i}$, for $i=1,2,3,4$.

to the buyer. However, if the payment time is shorter than the lead time, it will bring additional interests income and opportunity costs to the buyer due to the rest of stocks. After computing and comparing the results in Table 1 , we have found that the optimal profits will occur in $\operatorname{EJTP}_{1}\left(Q_{1}, n_{1}\right)$ under the manufacturer's production rate being 1300 units per year. Also, the worst profit will occur in $\operatorname{EJTP}_{4}\left(Q_{4}, n_{4}\right)$ under the manufacturer's production rate being 1100 units per year.

5.1.2. The Permissible Time $X$ and EJTP. In Section 5.1.2, we changed the retailer's purchasing cost to observe the value of profit; the solution results are illustrated in Figures 7 and 8, and the detailed result is shown in Table 2.

From Table 2, we have found that the optimal profits will occur in $\operatorname{EJTP}_{1}\left(Q_{1}, n_{1}\right)$ under the manufacturer's production rate being 1300 units per year which is the same as in Section 5.1.1. Also, the worst profit will occur in $\operatorname{EJTP}_{4}\left(Q_{4}, n_{4}\right)$ under the manufacturer's production rate being 1100 units per year.

5.2. The Variation under Different Z. In Section 5.2, we supposed that the maximum of defective rate is 0.3 . The manufacturer can change the production rate under any condition; also, the extra payment by changing the rate is ignored.
5.2.1. The Permissible Period $X$ and EJTP. We have changed manufacturer's purchasing cost $C_{m}$ by 0.5 per unit. In order to compare which condition is more beneficial, we formed detailed results in Table 3 . The solution results are illustrated in Figures 9 and 10.

From Table 3, we have found that the optimal profits will occur in $\operatorname{EJTP}_{1}\left(Q_{1}, n_{1}\right)$ under the manufacturer's defective rate being 0.1. Also, the worst profits will occur in $\operatorname{EJTP}_{4}\left(Q_{4}, n_{4}\right)$ under the manufacturer's defective rate being 0.3 .

5.2.2. The Permissible Period $Y$ and EJTP. We have changed retailer's purchasing costs $C_{r}$ by 0.5 per unit. In order to know which condition is more beneficial, we formed detailed results in Table 4. The solution results are illustrated in Figures 11 and 12.

From Table 4, we have found the optimal profits will occur in $\operatorname{EJTP}_{1}\left(Q_{1}, n_{1}\right)$ under the manufacturer's defective rate being 0.1 . Also, the worst profits will occur in $\operatorname{EJTP}_{4}\left(Q_{4}, n_{4}\right)$ under the manufacturer's defective rate being 0.3 .

5.3. Observation (See Figures 5-12 and Tables 1-4). In Section 5.1, we observed the variation of quantity per delivery, numbers of delivery, and EJTP by changing manufacturer's 
TABLE 2: The value of profit in different condition by changing $C_{r}$.

\begin{tabular}{|c|c|c|c|}
\hline & $P=1100$ & $P=1200$ & $P=1300$ \\
\hline$C_{r}$ & $240.0 \sim 245.0$ & $240.0 \sim 245.0$ & $240.0 \sim 245.0$ \\
\hline$n_{1}$ & 2 & 2 & 2 \\
\hline$Q_{1}$ & ${ }^{* *} 102.29$ & ${ }^{* * *} 103.49$ & ${ }^{* * * * *} 104.54$ \\
\hline $\mathrm{EJTP}_{1}$ & $59935.40 \sim 61249.25$ & $60082.94 \sim 61396.66$ & ${ }^{*} 60209.70 \sim 61523.33$ \\
\hline$\overline{C_{r}}$ & $245.5 \sim 250.0$ & $245.5 \sim 250.0$ & $245.5 \sim 250.0$ \\
\hline$n_{2}$ & 1 & 1 & 1 \\
\hline$Q_{2}$ & $576.70 \sim 566.45$ & $592.02 \sim 581.48$ & $605.98 \sim 595.17$ \\
\hline $\mathrm{EJTP}_{2}$ & $33700.94 \sim 35468.36$ & $34659.34 \sim 36408.84$ & $35488.95 \sim 37224.54$ \\
\hline$C_{r}$ & $240.0 \sim 245.0$ & $240.0 \sim 245.0$ & $240.0 \sim 245.0$ \\
\hline$n_{3}$ & 4 & 4 & 4 \\
\hline$Q_{3}$ & $289.19 \sim 226.66$ & $292.34 \sim 229.13$ & $295.07 \sim 231.27$ \\
\hline $\mathrm{EJTP}_{3}$ & $34255.30 \sim 42211.53$ & $34641.54 \sim 42514.20$ & $34971.60 \sim 42772.87$ \\
\hline$\overline{C_{r}}$ & $245.5 \sim 250.0$ & $245.5 \sim 250.0$ & $245.5 \sim 250.0$ \\
\hline$n_{4}$ & $\begin{array}{l}2\left(C_{r}=245.5 \sim 246.5\right) \\
1\left(C_{r}=247.0 \sim 250.0\right)\end{array}$ & $\begin{array}{l}2\left(C_{r}=245.5 \sim 246.0\right) \\
1\left(C_{r}=235.5 \sim 250.0\right)\end{array}$ & $\begin{array}{c}2\left(C_{r}=245.5\right) \\
1\left(C_{r}=236.0 \sim 250.0\right)\end{array}$ \\
\hline$Q_{4}$ & $\begin{array}{l}615.74 \sim 598.22\left(C_{r}=245.5 \sim 246.5\right) \\
775.30 \sim 663.75\left(C_{r}=247.0 \sim 250.0\right)\end{array}$ & $\begin{array}{c}627.23 \sim 618.37 \\
\left(C_{r}=245.5 \sim 246.0\right) \\
813.38 \sim 681.35\left(C_{r}=246.5 \sim 250\right)\end{array}$ & $\begin{array}{c}637.48\left(C_{r}=245.5\right) \\
850.10 \sim 697.38\left(C_{r}=246 \sim 250\right)\end{array}$ \\
\hline $\mathrm{EJTP}_{4}$ & 20215.40 29779.79 & $21168.25 \sim 30881.82$ & $21988.73 \sim 31837.60$ \\
\hline
\end{tabular}

${ }^{*}$ Optimal solution of $\mathrm{EJTP}_{i}$.

**,***,*** We cannot observe the variation because of low increasing rate; in fact, $Q_{1}$ will decrease slightly when $C_{r}$ increases.

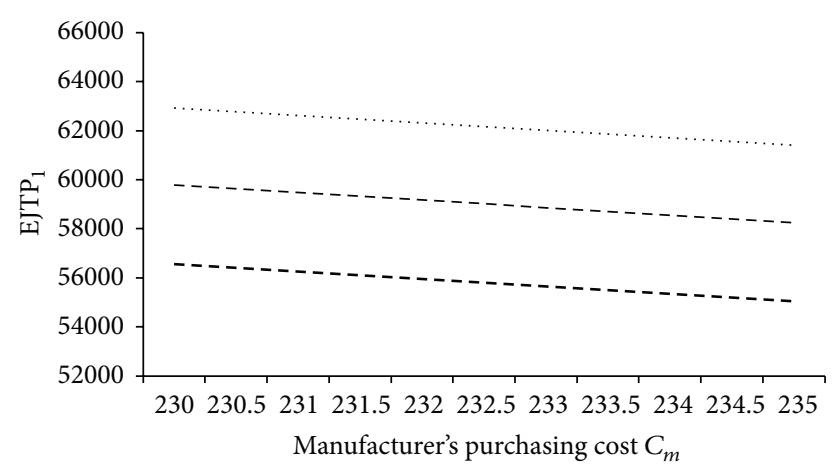

(a) The value of $\mathrm{EJTP}_{1}$ by changing $C_{m}$ under different $Z$

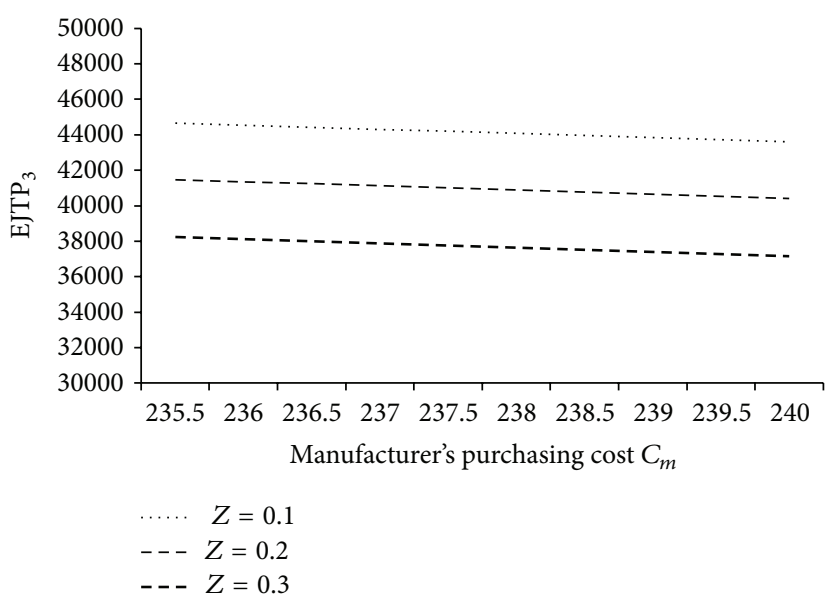

(c) The value of $\mathrm{EJTP}_{3}$ by changing $C_{m}$ under different $Z$

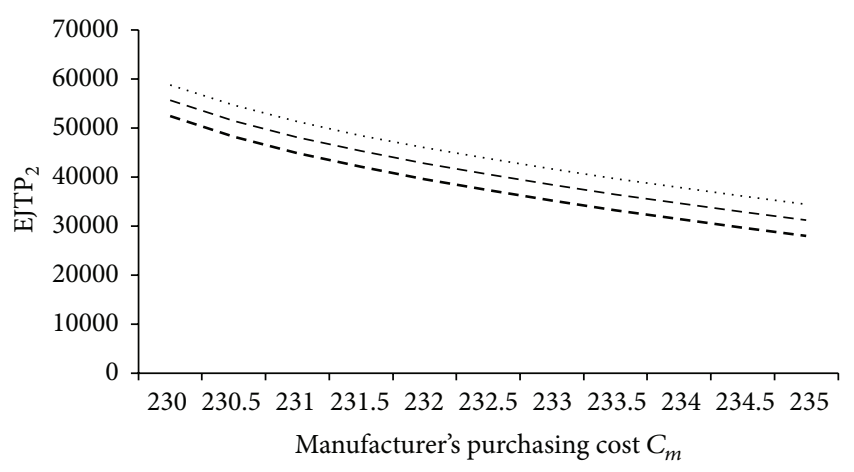

(b) The value of $\mathrm{EJTP}_{2}$ by changing $C_{m}$ under different $Z$

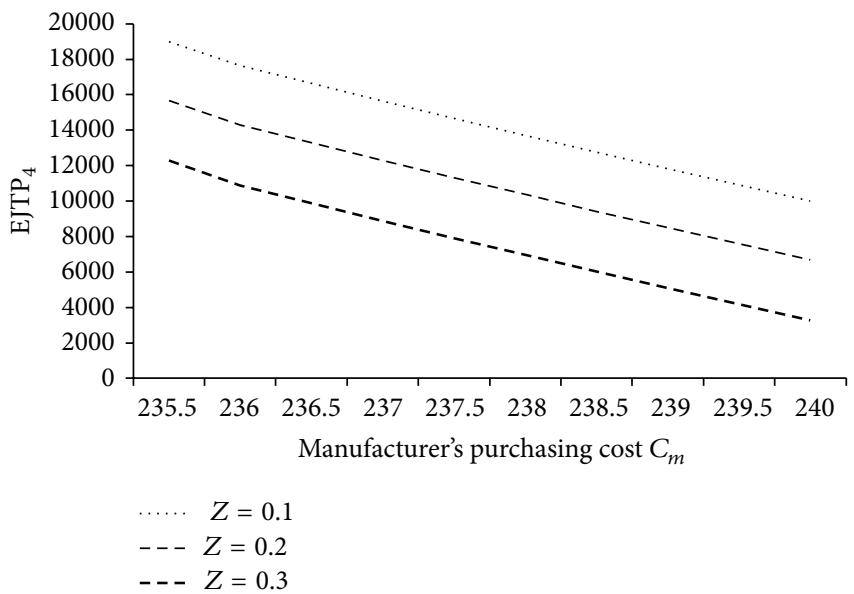

(d) The value of $\mathrm{EJTP}_{4}$ by changing $C_{m}$ under different $Z$

FIGURE 10: The value of profit by changing $C_{m}$ in $\mathrm{EJTP}_{i}$, for $i=1,2,3,4$. 


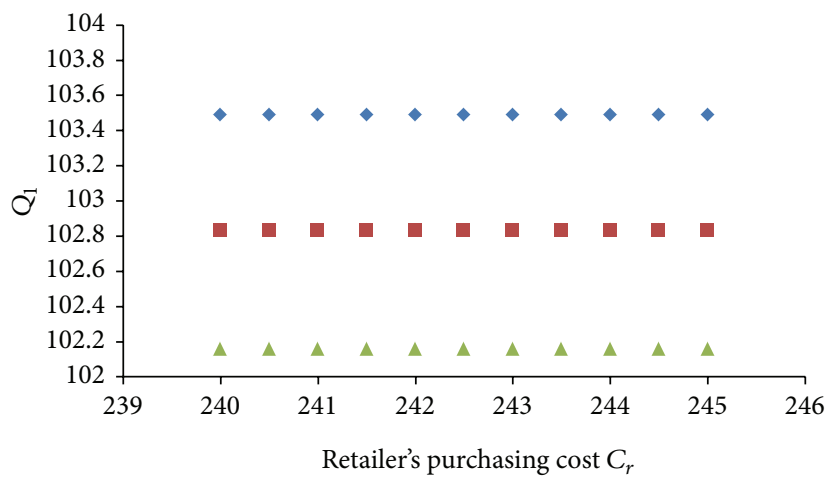

(a) The value of $Q_{1}$ by changing $C_{r}$ under different $Z$

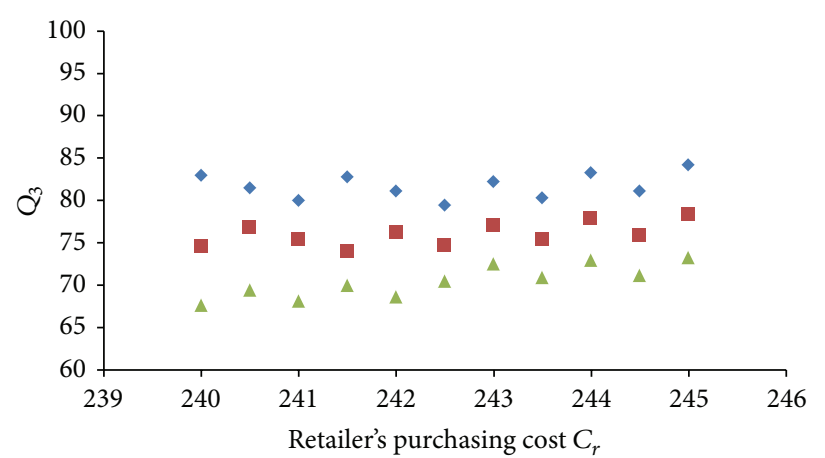

$\checkmark=0.1$

- $Z=0.2$

$\triangle Z=0.3$

(c) The value of $Q_{3}$ by changing $C_{r}$ under different $Z$

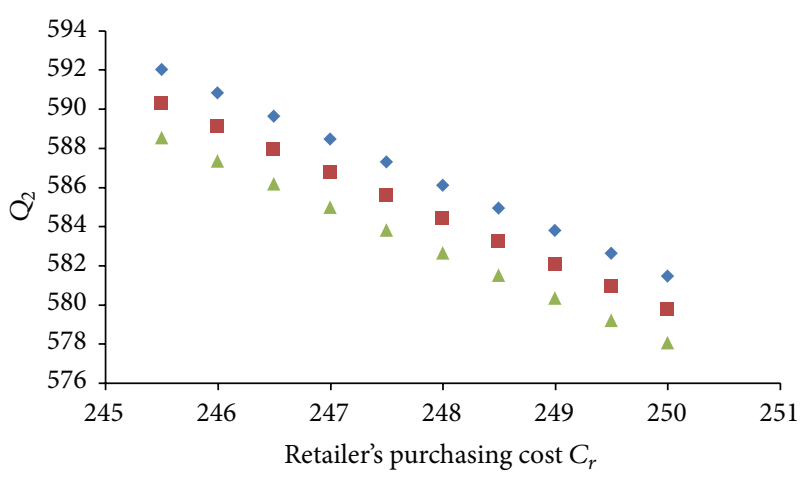

(b) The value of $Q_{2}$ by changing $C_{r}$ under different $Z$

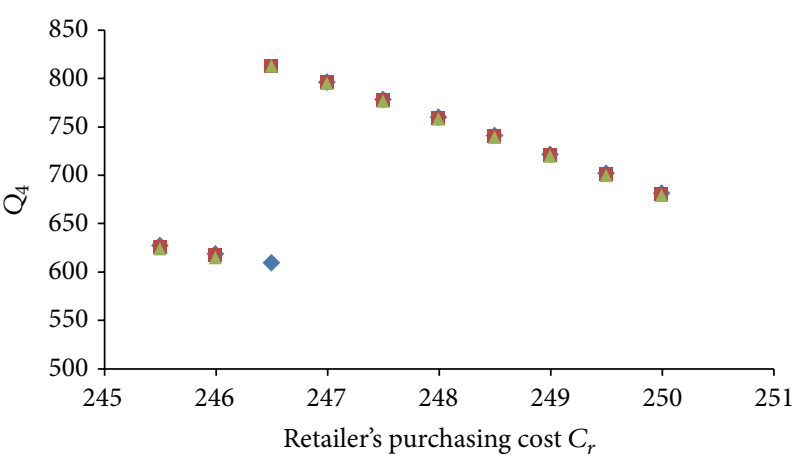

$\checkmark Z=0.1$

- $Z=0.2$

$\triangle Z=0.3$

(d) The value of $Q_{4}$ by changing $C_{r}$ under different $Z$

FIGURE 11: The value of delivery quantity by changing $C_{r}$ in $Q_{i}$, for $i=1,2,3,4$.

TABLE 3: The value of profit in different condition by changing $C_{m}$.

\begin{tabular}{|c|c|c|c|}
\hline & $Z=0.1$ & $Z=0.2$ & $Z=0.3$ \\
\hline$C_{m}$ & $230.0 \sim 235.0$ & $230.0 \sim 235.0$ & $230.0 \sim 235.0$ \\
\hline$n_{1}$ & 2 & 2 & 2 \\
\hline$Q_{1}$ & $103.39 \sim 103.49$ & $102.73 \sim 102.83$ & $102.06 \sim 102.16$ \\
\hline $\mathrm{EJTP}_{1}$ & ${ }^{*} 62930.18 \sim 61396.67$ & $59783.53 \sim 58250.30$ & 56571.47 55038.52 \\
\hline$C_{m}$ & $230.0 \sim 235.0$ & $230.0 \sim 235.0$ & $230.0 \sim 235.0$ \\
\hline$n_{2}$ & 1 & 1 & 1 \\
\hline$Q_{2}$ & $194.04 \sim 593.21$ & $193.48 \sim 591.50$ & $192.90 \sim 589.73$ \\
\hline $\mathrm{EJTP}_{2}$ & $58779.07 \sim 34462.59$ & 55616.48 31264.91 & $52388.27 \sim 28000.05$ \\
\hline$C_{m}$ & $235.5 \sim 240$ & $235.5 \sim 240$ & $235.5 \sim 240$ \\
\hline$n_{3}$ & 13 & 14 & 15 \\
\hline$Q_{3}$ & $84.04 \sim 82.95$ & $78.23 \sim 77.23$ & $73.12 \sim 72.29$ \\
\hline $\mathrm{EJTP}_{3}$ & $44660.66 \sim 43599.22$ & $41473.30 \sim 40411.53$ & $38226.15 \sim 37164.46$ \\
\hline$\overline{C_{m}}$ & $235.5 \sim 240$ & $235.5 \sim 240$ & $235.5 \sim 240$ \\
\hline$n_{4}$ & $\begin{array}{c}2\left(C_{m}=235.5\right) \\
1\left(C_{m}=236 \sim 240\right)\end{array}$ & $\begin{array}{c}2\left(C_{m}=235.5\right) \\
1\left(C_{m}=236 \sim 240\right)\end{array}$ & $\begin{array}{c}2\left(C_{m}=235.5\right) \\
1\left(C_{m}=236 \sim 240\right)\end{array}$ \\
\hline$Q_{4}$ & $\begin{array}{c}653.70\left(C_{m}=235.5\right) \\
897.88 \sim 1022.77\left(C_{m}=236 \sim 240\right)\end{array}$ & $\begin{array}{c}652.03\left(C_{m}=235.5\right) \\
897.51 \sim 1021.71\left(C_{m}=236 \sim 240\right)\end{array}$ & $\begin{array}{c}650.29\left(C_{m}=235.5\right) \\
897.08 \sim 1020.58\left(C_{m}=236 \sim 240\right)\end{array}$ \\
\hline $\mathrm{EJTP}_{4}$ & $19000.21 \sim 10007.45$ & $15675.17 \sim 6673.83$ & $12278.57 \sim 3629.20$ \\
\hline
\end{tabular}

${ }^{*}$ Optimal solution of $\mathrm{EJTP}_{i}$. 


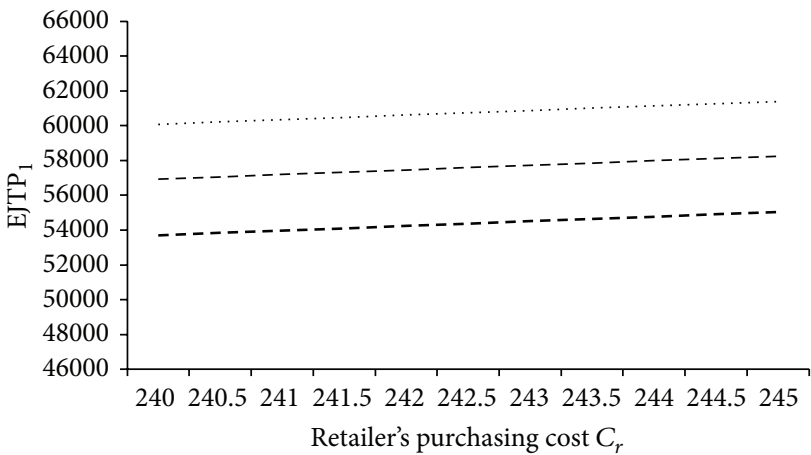

(a) The value of $\mathrm{EJTP}_{1}$ by changing $C_{r}$ under different $Z$

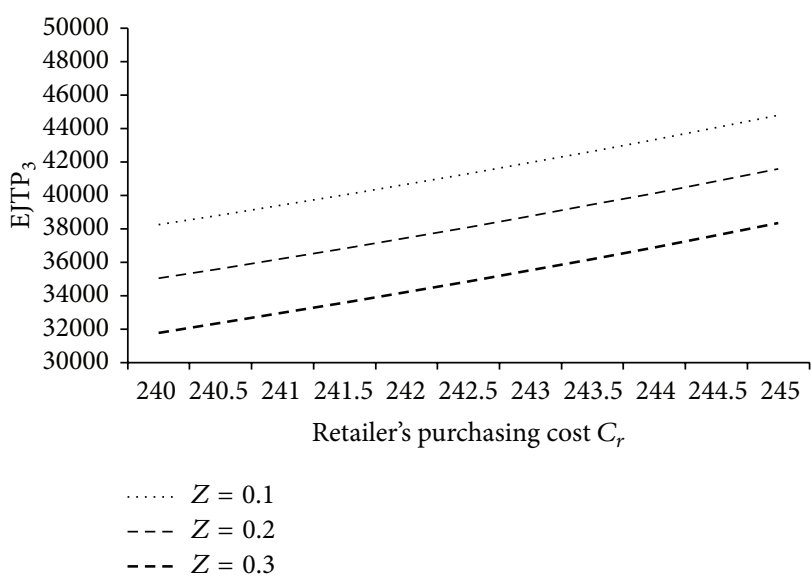

(c) The value of $\mathrm{EJTP}_{3}$ by changing $C_{r}$ under different $Z$

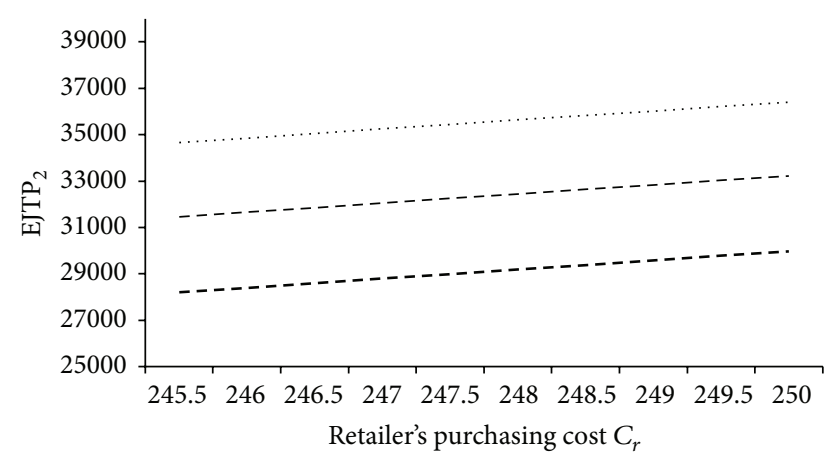

(b) The value of $\mathrm{EJTP}_{2}$ by changing $C_{r}$ under different $Z$

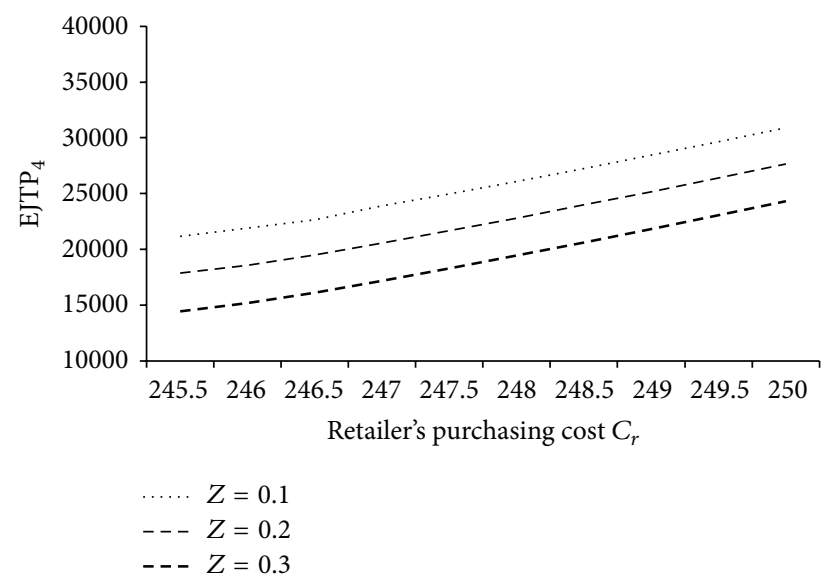

(d) The value of $\mathrm{EJTP}_{4}$ by changing $C_{r}$ under different $Z$

FIGURE 12: The value of profit by changing $C_{r}$ in $\mathrm{EJTP}_{i}$, for $i=1,2,3,4$.

purchasing costs $C_{m}$ or retailer's purchasing costs $C_{r}$ under different production rate. Obviously, higher production rate will yield higher profits. All EJTP of each case decreases when $C_{m}$ increases. In Section 5.1.1, the optimal profits occur in $\operatorname{EJTP}_{1}\left(Q_{1}, n_{1}\right)$ under $P=1300$; in Section 5.1.2, the optimal profits also occur in $\operatorname{EJTP}_{1}\left(Q_{1}, n_{1}\right)$ under $P=1300$.

In Section 5.2, the observations are shown under different defective rate consideration. Surely, higher defective rate leads manufacturer to pay more costs to rework defective items and deal with scrap. As $C_{m}$ increases, all EJTP of each case decreases; nevertheless, increasing $\mathrm{C}_{r}$ brings decreasing EJTP contrarily. In Section 5.2.1, the optimal profits occur in $\operatorname{EJTP}_{1}\left(Q_{1}, n_{1}\right)$ under $Z=0.1$; in Section 5.2.2, the optimal profits also occur in $\operatorname{EJTP}_{1}\left(Q_{1}, n_{1}\right)$ under $Z=0.1$.

Because of the relationship between the price and payment period, the decision-makers can get different payment period by varying the price. When the supply chain is successfully integrated, this variation can lead to unnecessary costs reduction or enhance the performance.

\section{Conclusions and Future Works}

The main purpose of every firm is to maximize profits. There are two ways to enhance profits: one is to raise the products' selling price, and the other is to lower the relevant costs in supply chain. To raise the products' selling price, firms have to enhance products' quality and show uniqueness to convince customers. Alternatively, firms can provide proper strategies to reduce relevant costs such as purchasing costs, production costs, holding costs, and transportation costs.

Permissible delay in payments is a common commercial strategy in real business transactions, since the purpose of business strategies is to enhance the flexibility of capital. In other words, firms can obtain additional interests income from sales revenue during the payment period; yet, upstream firms simply grant loans to downstream firms without any interests. Thus, it is of great importance to decide the length of payment period in an SCM setting. There are many ways to balance the costs or revenue of each firm. From the reward perspective, providing discounts is a direct way to attract downstream firms in accepting shorter payment period. On the other hand, which is from the punishment perspective, downstream firms must pay extra costs if they wish to enjoy a longer payment period. Whether it is from the rewards or the punishments perspective, the purpose is always about shortening the payment period. In this paper, we have used different ways to determine the payment period. We set the relationship of purchasing costs and payment period as inverse proportion; that is, payment period is floating and higher purchasing costs will bring shorter payment period. From the results in Section 5, decision-makers should negotiate with their upstream or downstream firms to enhance supply chain performance. From the supplier and manufacturer's 
TABLE 4: The value of profit in different condition by changing $C_{r}$.

\begin{tabular}{|c|c|c|c|}
\hline & $Z=0.1$ & $Z=0.2$ & $Z=0.3$ \\
\hline$C_{r}$ & $240.0 \sim 245.0$ & $240.0 \sim 245.0$ & $240.0 \sim 245.0$ \\
\hline$n_{1}$ & 2 & 2 & 2 \\
\hline$Q_{1}$ & *103.49 & ${ }^{* * *} 102.83$ & ${ }^{* * *} 102.16$ \\
\hline $\mathrm{EJTP}_{1}$ & *60082.94 61396.67 & $56923.45 \sim 58250.30$ & $53698.28 \sim 55038.52$ \\
\hline$\overline{C_{r}}$ & $245.5 \sim 250$ & $245.5 \sim 250$ & $245.5 \sim 250$ \\
\hline$n_{2}$ & 1 & 1 & 1 \\
\hline$Q_{2}$ & $592.02 \sim 581.48$ & $590.31 \sim 579.80$ & $588.54 \sim 578.07$ \\
\hline $\mathrm{EJTP}_{2}$ & $34658.46 \sim 36408.84$ & $31462.16 \sim 33225.06$ & $28198.72 \sim 29974.40$ \\
\hline$C_{r}$ & $240.0 \sim 245$ & $240.0 \sim 245$ & $240.0 \sim 245$ \\
\hline$n_{3}$ & $\begin{array}{c}17\left(C_{r}=240 \sim 241\right) \\
16\left(C_{r}=241.5 \sim 242.5\right) \\
15\left(C_{r}=243 \sim 243.5\right) \\
14\left(C_{r}=244 \sim 244.5\right) \\
13\left(C_{r}=245\right)\end{array}$ & $\begin{array}{c}19\left(C_{r}=240\right) \\
18\left(C_{r}=240.5 \sim 241.5\right) \\
17\left(C_{r}=242 \sim 242.5\right) \\
16\left(C_{r}=243 \sim 243.5\right) \\
15\left(C_{r}=244 \sim 244.5\right) \\
14\left(C_{r}=245\right)\end{array}$ & $\begin{array}{c}21\left(C_{r}=240\right) \\
20\left(C_{r}=240.5 \sim 241\right) \\
19\left(C_{r}=241.5 \sim 242\right) \\
18\left(C_{r}=242.5\right) \\
17\left(C_{r}=243 \sim 243.5\right) \\
16\left(C_{r}=244 \sim 244.5\right) \\
15\left(C_{r}=245\right)\end{array}$ \\
\hline$Q_{3}$ & $\begin{array}{c}82.95 \sim 79.97\left(C_{r}=240 \sim 241\right) \\
82.77 \sim 81.1\left(C_{r}=241.5 \sim 242.5\right) \\
82.21 \sim 80.32\left(C_{r}=243 \sim 243.5\right) \\
83.25 \sim 81.12\left(C_{r}=244 \sim 244.5\right) \\
84.17\left(C_{r}=245\right)\end{array}$ & $\begin{array}{c}74.53\left(C_{r}=240\right) \\
76.84 \sim 73.99\left(C_{r}=240.5 \sim 241.5\right) \\
76.29 \sim 74.71\left(C_{r}=242 \sim 242.5\right) \\
77.09 \sim 75.33\left(C_{r}=243 \sim 243.5\right) \\
75.82\left(C_{r}=244 \sim 244.5\right) \\
78.34\left(C_{r}=245\right)\end{array}$ & $\begin{array}{c}67.62\left(C_{r}=240\right) \\
69.40 \sim 68.14\left(C_{r}=240.5 \sim 241\right) \\
69.98 \sim 68.60\left(C_{r}=241.5 \sim 242\right) \\
70.48\left(C_{r}=242.5\right) \\
72.52 \sim 70.88\left(C_{r}=243 \sim 243.5\right) \\
72.96 \sim 71.13\left(C_{r}=244 \sim 244.5\right) \\
73.23\left(C_{r}=245\right)\end{array}$ \\
\hline $\mathrm{EJTP}_{3}$ & $38237.07 \sim 44777.73$ & $35037.87 \sim 41590.40$ & $31785.46 \sim 38343.24$ \\
\hline$C_{r}$ & $245.5 \sim 250$ & $245.5 \sim 250$ & $245.5 \sim 250$ \\
\hline$n_{4}$ & $\begin{array}{c}2\left(C_{r}=245.5\right) \\
1\left(C_{r}=246 \sim 250\right)\end{array}$ & $\begin{array}{l}2\left(C_{r}=245.5 \sim 246\right) \\
1\left(C_{r}=246.5 \sim 250\right)\end{array}$ & $\begin{array}{l}2\left(C_{r}=245.5 \sim 246\right) \\
1\left(C_{r}=246.5 \sim 250\right)\end{array}$ \\
\hline$Q_{4}$ & $\begin{array}{c}627.23\left(C_{r}=245.5\right) \\
618.37 \sim 681.35\left(C_{r}=246 \sim 250\right)\end{array}$ & $\begin{array}{l}625.65 \sim 616.76\left(C_{r}=245.5\right) \\
246.5 \sim 250\left(C_{r}=246 \sim 250\right)\end{array}$ & $\begin{array}{c}624.00 \sim 615.08\left(C_{r}=245.5 \sim 246\right) \\
812.57 \sim 679.24\left(C_{r}=246 \sim 250\right)\end{array}$ \\
\hline $\mathrm{EJTP}_{4}$ & $21168.36 \sim 30881.82$ & $17850.43 \sim 27637.25$ & $14461.21 \sim 24324.25$ \\
\hline
\end{tabular}

${ }^{*}$ Optimal solution of $\mathrm{EJTP}_{i}$.

${ }_{* *, * * *, * * *}$ We cannot observe the variation because of low increasing rate; in fact, $Q_{1}$ will decrease slightly when $C_{r}$ increases.

viewpoint, EJTP moves up when the purchasing costs of manufacturer go down. However, there is a contrary result on the manufacturer and supplier's side. Higher purchasing costs of the supplier will lead to lower profits. Decision-makers should know where their firms are positioned in the supply chain and may thus make appropriate decisions.

Defective rate is also an important factor in the manufacturing process. The higher the probability of defective product occurrence, the higher the cost and more time will be spent by the manufacturer; these may include reordering the materials and reproducing, repairing, and declaring the scrap. Additionally, defective rate is one of the direct factors to affect the amount of storage. If retailers do not have enough stocks to satisfy customers' needs, customers may lose their patience and therefore choose other retailers. Surely, it is important to accurately grasp the situation of production lines.

From what has been discussed above, we developed a three-echelon inventory model to determine optimal joint total profits. Firstly, we have developed four inventory models in Section 3 according to different permissible delay payment period and lead time. Secondly, we computed the decision variables, economical delivery quantity, and the number of deliveries per production run from the manufacturer to the retailer. Finally, we observed and found the optimal profits by varying the manufacturer's purchasing costs or the supplier's purchasing costs.

Compared with Yang and Tseng's [14] article, although they considered the defective products to occur in the three echelons, we only assumed the defective products occur in the manufacturing process. In this paper, we also focused on the relationship between materials/finished product's sale price and the permissible delay period. We assumed that the relationship is inverse proportion and developed the function while Yang and Tseng's [14] simply focused on variable lead time and assumed that the permissible delay period is constant.

In the future, we can add more conditions or assumptions such as ignoring the backorder and variable lead time which were considered by Yang and Tseng's [14]. The assumptions can be added again to develop more practical inventory models. Besides, multiple sellers or multiple purchasers are 
not unusual situations in commerce. Moreover, the parameters in this paper are fixed while some of them (such as demand or defective rate) may be unfixed in practice by using fuzzy theory. The fuzzy variables can lead to better results. The issue regarding deteriorating products is worthy of deliberation in the inventory model since all products would face deterioration (i.e., rust or decay) sooner or later. We look forward to illustrating real-world numerical exam.

\section{Conflict of Interests}

The authors declare that there is no conflict of interests regarding the publication of this paper.

\section{References}

[1] R. Fogarty, "Ten ways to integrate curriculum," Educational Leadership, vol. 49, no. 2, pp. 61-65, 1991.

[2] R. Frankel, "The role and relevance of refocused inventory: supply chain management solutions," Business Horizons, vol. 49, no. 4, pp. 275-286, 2006.

[3] M. Ben-Daya, R. As'Ad, and M. Seliaman, "An integrated production inventory model with raw material replenishment considerations in a three layer supply chain," International Journal of Production Economics, vol. 143, no. 1, pp. 53-61, 2013.

[4] H. M. Beheshti, "A decision support system for improving performance of inventory management in a supply chain network," International Journal of Productivity and Performance Management, vol. 59, no. 5, pp. 452-467, 2010.

[5] F. W. Harris, "How many parts to make at once," Operations Research, vol. 38, no. 6, pp. 947-950, 1913.

[6] S. K. Goyal, "An integrated inventory model for a single supplier-single customer problem," International Journal of Production Research, vol. 15, no. 1, pp. 107-111, 1977.

[7] W. Jammernegg and G. Reiner, "Performance improvement of supply chain processes by coordinated inventory and capacity management," International Journal of Production Economics, vol. 108, no. 1-2, pp. 183-190, 2007.

[8] D. L. Olson and M. Xie, "A comparison of coordinated supply chain inventory management systems," International Journal of Services and Operations Management, vol. 6, no. 1, pp. 73-88, 2010.

[9] Y. Huang, G. Q. Huang, and S. T. Newman, "Coordinating pricing and inventory decisions in a multi-level supply chain: a game-theoretic approach," Transportation Research Part E: Logistics and Transportation Review, vol. 47, no. 2, pp. 115-129, 2011.

[10] I. Giannoccaro and P. Pontrandolfo, "Inventory management in supply chains: a reinforcement learning approach," International Journal of Production Economics, vol. 78, no. 2, pp. 153$161,2002$.

[11] L. E. Cárdenas-Barrón, J.-T. Teng, G. Treviño-Garza, H.-M. Wee, and K.-R. Lou, "An improved algorithm and solution on an integrated production-inventory model in a three-layer supply chain," International Journal of Production Economics, vol. 136, no. 2, pp. 384-388, 2012.

[12] S. S. Sana, "A production-inventory model of imperfect quality products in a three-layer supply chain," Decision Support Systems, vol. 50, no. 2, pp. 539-547, 2011.
[13] K.-J. Chung, L. Eduardo Cárdenas-Barrón, and P.-S. Ting, "An inventory model with non-instantaneous receipt and exponentially deteriorating items for an integrated three layer supply chain system under two levels of trade credit," International Journal of Production Economics, vol. 155, pp. 310-317, 2014.

[14] M. F. Yang and W. C. Tseng, “Three-echelon inventory model with permissible delay in payments under controllable lead time and backorder consideration," Mathematical Problems in Engineering, vol. 2014, Article ID 809149, 16 pages, 2014.

[15] M. A. Rad, F. Khoshalhan, and C. H. Glock, "Optimizing inventory and sales decisions in a two-stage supply chain with imperfect production and backorders," Computers \& Industrial Engineering, vol. 74, pp. 219-227, 2014.

[16] R. L. Schwaller, "EOQ under inspection costs," Production and Inventory Management Journal, vol. 29, no. 3, pp. 22-24, 1988.

[17] M. J. Paknejad, F. Nasri, and J. F. Affisco, "Defective units in a continuous review (s, Q) system," International Journal of Production Research, vol. 33, no. 10, pp. 2767-2777, 1995.

[18] J. J. Liu and P. Yang, "Optimal lot-sizing in an imperfect production system with homogeneous reworkable jobs," European Journal of Operational Research, vol. 91, no. 3, pp. 517-527, 1996.

[19] M. K. Salameh and M. Y. Jaber, "Economic production quantity model for items with imperfect quality," International Journal of Production Economics, vol. 64, no. 1, pp. 59-64, 2000.

[20] A. Eroglu and G. Ozdemir, "An economic order quantity model with defective items and shortages," International Journal of Production Economics, vol. 106, no. 2, pp. 544-549, 2007.

[21] B. Pal, S. S. Sana, and K. Chaudhuri, "Three-layer supply chain-a production-inventory model for reworkable items," Applied Mathematics and Computation, vol. 219, no. 2, pp. 530543, 2012.

[22] B. Sarkar, L. E. Cárdenas-Barrón, M. Sarkar, and M. L. Singgih, "An economic production quantity model with random defective rate, rework process and backorders for a single stage production system," Journal of Manufacturing Systems, vol. 33, no. 3, pp. 423-435, 2014.

[23] L. E. Cárdenas-Barrón, "Economic production quantity with rework process at a single-stage manufacturing system with planned backorders," Computers and Industrial Engineering, vol. 57, no. 3, pp. 1105-1113, 2009.

[24] H. N. Soni and K. A. Patel, "Optimal strategy for an integrated inventory system involving variable production and defective items under retailer partial trade credit policy," Decision Support Systems, vol. 54, no. 1, pp. 235-247, 2012.

[25] S. K. Goyal, "Economic order quantity under conditions of permissible delay in payments," Journal of the Operational Research Society, vol. 36, no. 4, pp. 335-338, 1985.

[26] S. P. Aggarwal and C. K. Jaggi, "Ordering policies of deteriorating items under permissible delay in payments," Journal of the Operational Research Society, vol. 46, pp. 658-662, 1995.

[27] A. M. M. Jamal, B. R. Sarker, and S. Wang, "An ordering policy for deteriorating items with allowable shortage and permissible delay in payment," Journal of the Operational Research Society, vol. 48 , no. 8 , pp. 826-833, 1997.

[28] J.-T. Teng, "On the economic order quantity under conditions of permissible delay in payments," Journal of the Operational Research Society, vol. 53, no. 8, pp. 915-918, 2002.

[29] C. K. Huang, D. M. Tsai, J. C. Wu, and K. J. Chung, "An integrated vendor-buyer inventory model with order-processing cost reduction and permissible delay in payments," European Journal of Operational Research, vol. 202, no. 2, pp. 473-478, 2010. 
[30] D. Ha and S.-L. Kim, "Implementation of JIT purchasing: an integrated approach," Production Planning \& Control: The Management of Operations, vol. 8, no. 2, pp. 152-157, 1997.

[31] K.-R. Lou and W.-C. Wang, "A comprehensive extension of an integrated inventory model with ordering cost reduction and permissible delay in payments," Applied Mathematical Modelling, vol. 37, no. 7, pp. 4709-4716, 2013.

[32] C.-K. Huang, "An integrated inventory model under conditions of order processing cost reduction and permissible delay in payments," Applied Mathematical Modelling: Simulation and Computation for Engineering and Environmental Systems, vol. 34, no. 5, pp. 1352-1359, 2010.

[33] J. Li, H. Feng, and Y. Zeng, "Inventory games with permissible delay in payments," European Journal of Operational Research, vol. 234, no. 3, pp. 694-700, 2014.

[34] A. Meca, J. Timmer, I. Garcia-Jurado, and P. Borm, "Inventory games," European Journal of Operational Research, vol. 156, no. 1, pp. 127-139, 2004.

[35] B. Sarkar, S. S. Sana, and K. Chaudhuri, "An inventory model with finite replenishment rate, trade credit policy and pricediscount offer," Journal of Industrial Engineering, vol. 2013, Article ID 672504, 18 pages, 2013. 


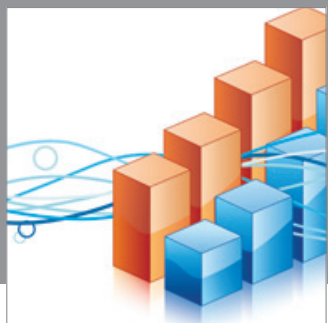

Advances in

Operations Research

mansans

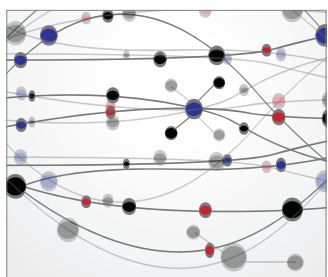

The Scientific World Journal
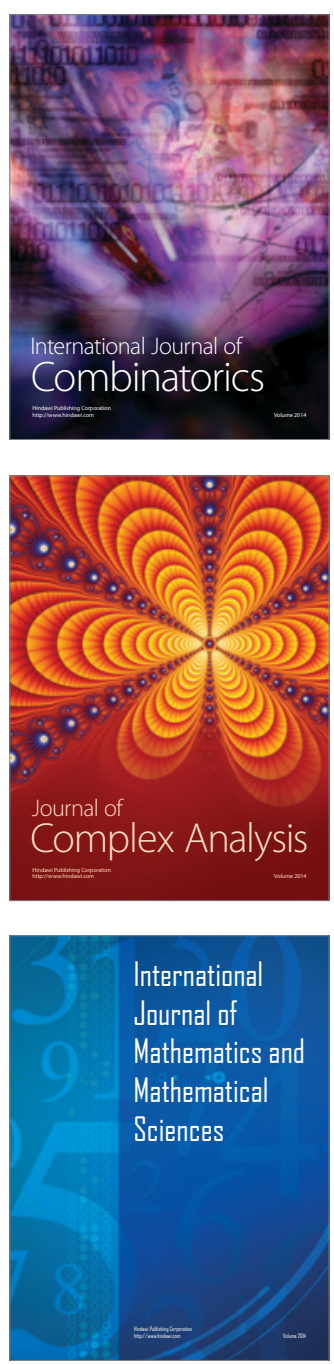
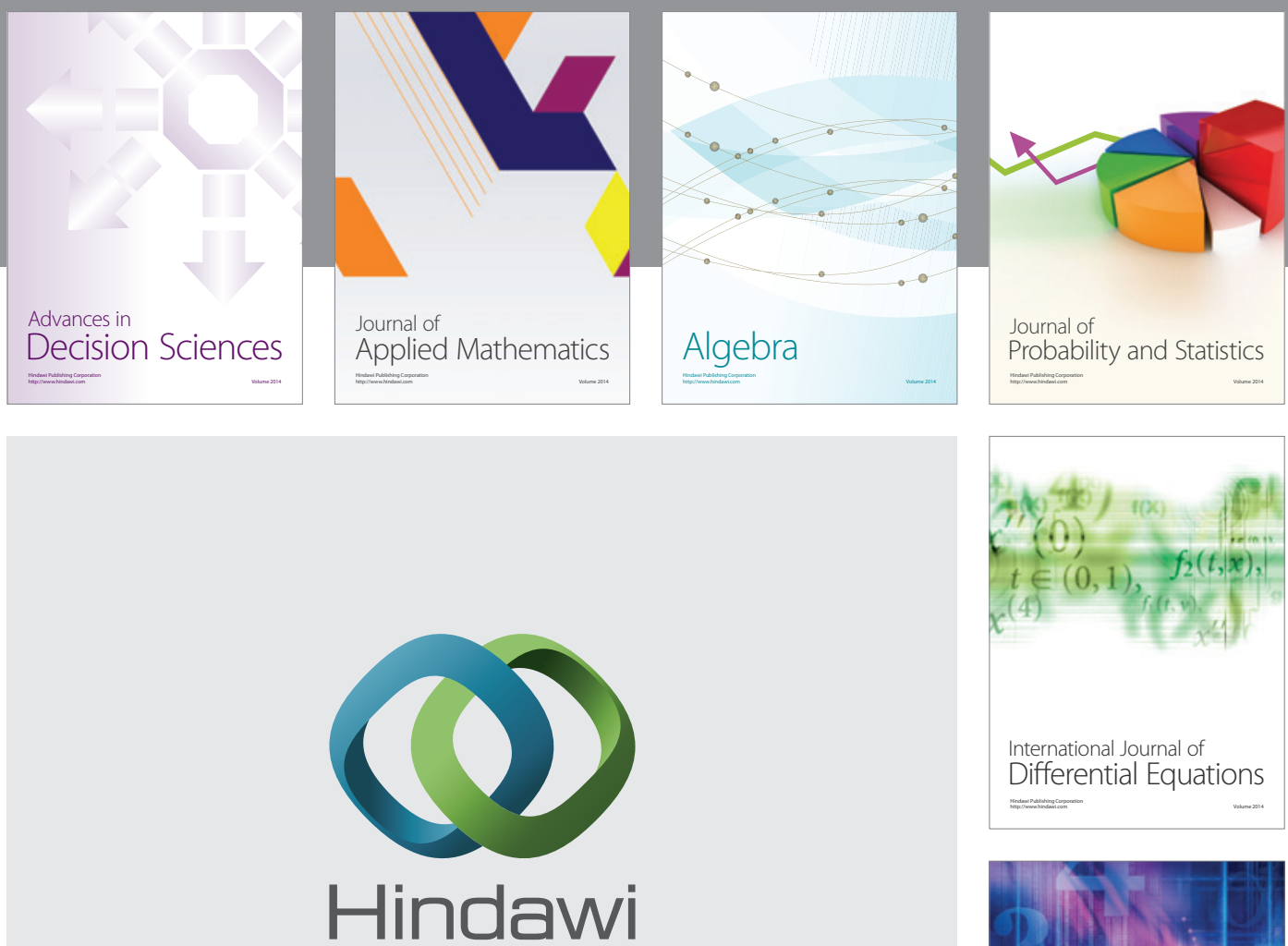

Submit your manuscripts at http://www.hindawi.com
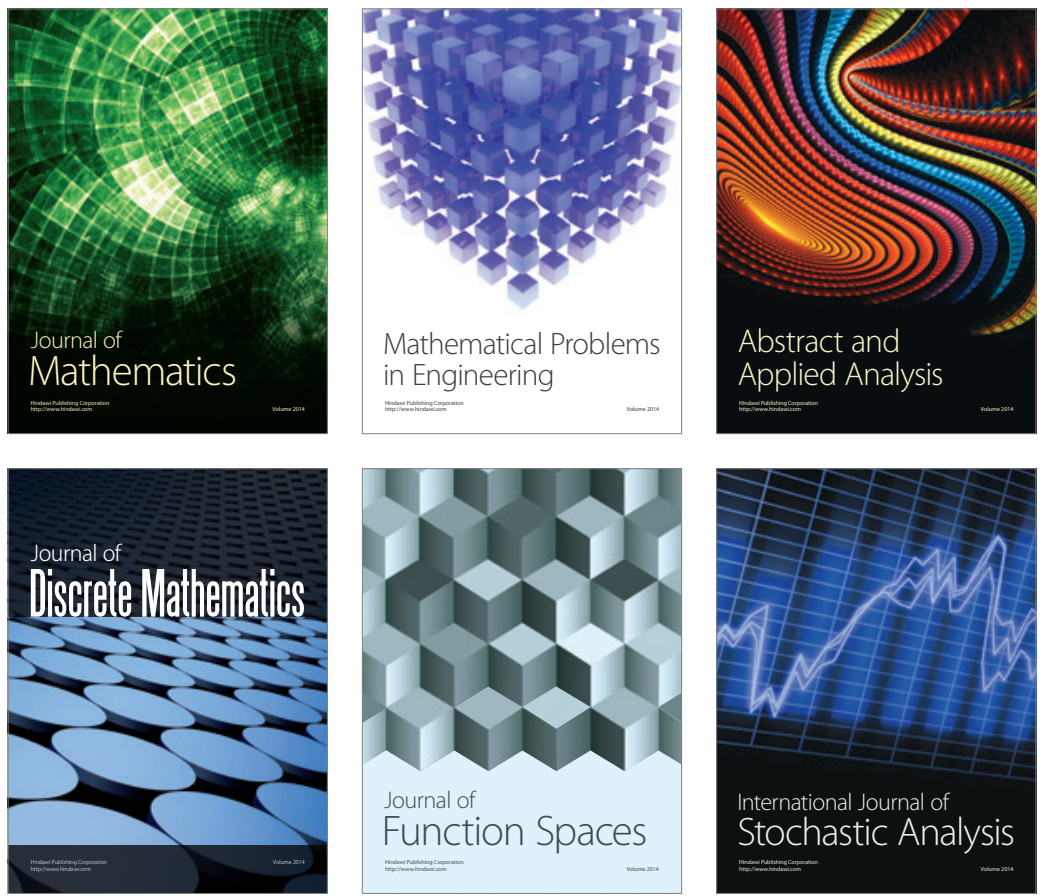

Journal of

Function Spaces

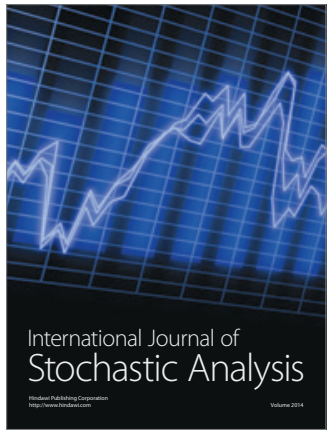

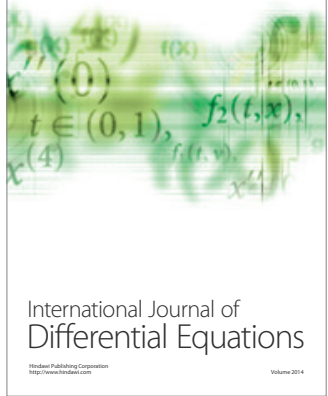
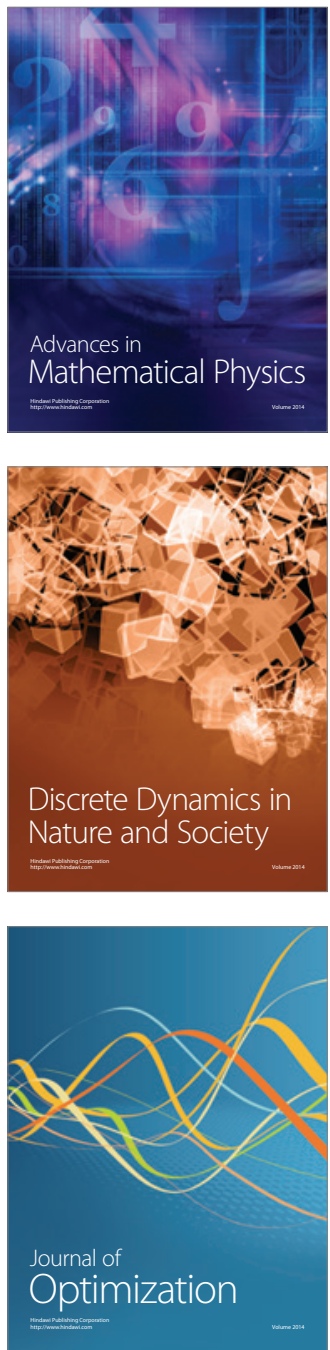